\title{
SELMER COMPANION CURVES
}

\author{
BARRY MAZUR AND KARL RUBIN
}

\begin{abstract}
We say that two elliptic curves $E_{1}, E_{2}$ over a number field $K$ are $n$-Selmer companions for a positive integer $n$ if for every quadratic character $\chi$ of $K$, there is an isomorphism $\operatorname{Sel}_{n}\left(E_{1}^{\chi} / K\right) \cong \operatorname{Sel}_{n}\left(E_{2}^{\chi} / K\right)$ between the $n$ Selmer groups of the quadratic twists $E_{1}^{\chi}, E_{2}^{\chi}$. We give sufficient conditions for two elliptic curves to be $n$-Selmer companions, and give a number of examples of non-isogenous pairs of companions.
\end{abstract}

\section{INTRODUCTION}

There has recently been much interest in the relative densities of $p$-Selmer ranks of elliptic curves in families consisting of all quadratic twists of a given elliptic curve $E$ over a fixed number field $K$. There are

- conjectures about these relative densities [19,

- some theorems for $p=2$ for elliptic curves over $K=\mathbf{Q}$ with full 2-torsion rational [7,28,

- some theorems for $p=2$ for elliptic curves over arbitrary number fields $K$ with other restrictions on the rational 2-torsion [10,11.

The results here are still fragmentary, but the general sense of these conjectures and results is that the relative densities of the sets of characters $\chi$ such that $E^{\chi}$ has a fixed $p$-Selmer rank (the characters being ordered appropriately) are expected to depend only on a few of the basic features of the elliptic curve $E$ and number field $K$. For example, if $p=2$ and $\operatorname{Gal}(K(E[2]) / K) \cong S_{3}$, then these densities depend only on a single parameter, a rational number $\delta(E, K) \in[0,1]$ that we call the disparity [11. Moreover, if $K$ has a real embedding, then $\delta(E, K)=1 / 2$, so for such $E$ and $K$ these densities are absolute constants.

At the present we have no results at all giving rank densities of $p$-Selmer groups of quadratic twist families when $p>2$. For example, fix a number field $K$, a prime number $p$, and an elliptic curve $E$ over $K$. We expect that the ranks of the $p$-Selmer groups in the family of all quadratic twists of $E$ over $K$ are unbounded, but we can prove this for no triple $(K, p, E)$ with $p>2$. Nonetheless, we have precise expectations [19] for the statistics governing $p$-rank densities in this context.

In view of this type of constancy of densities of Selmer ranks, let us consider the Selmer rank function itself, rather than its statistics, and formulate the following inverse question. For every positive integer $n$, let $\operatorname{Sel}_{n}(E / K)$ denote the $n$-Selmer group of $E$ over $K$ (see Definition 6.2), and for every quadratic character $\chi$ of $K$, we denote by $E^{\chi}$ the quadratic twist of $E$ by $\chi$.

Received by the editors August 18, 2012 and, in revised form, February 28, 2013.

2010 Mathematics Subject Classification. Primary 11G056; Secondary 11G40, 11 G07.

This material is based upon work supported by the National Science Foundation under grants DMS-1065904 and DMS-0968831. 
Question 1.1. For a fixed prime $p$ and number field $K$, how much information about $E$ can be read off from the function $\chi \mapsto \operatorname{dim}_{\mathbf{F}_{p}} \operatorname{Sel}_{p}\left(E^{\chi} / K\right)$ ?

This leads us to the following definition.

Definition 1.2. We will say that two elliptic curves $E_{1}, E_{2}$ over $K$ are $n$-Selmer companions if for every quadratic character $\chi$ of $K$ there is a group isomorphism

$$
\operatorname{Sel}_{n}\left(E_{1}^{\chi} / K\right) \cong \operatorname{Sel}_{n}\left(E_{2}^{\chi} / K\right) .
$$

If $n=\prod p_{i}^{k_{i}}$, then $E_{1}$ and $E_{2}$ are $n$-Selmer companions if and only if they are $p_{i}^{k_{i}}$-Selmer companions for every $i$. For this reason, in what follows we will restrict ourselves to the case where $n$ is a prime power.

If $E_{1}$ and $E_{2}$ are isogenous over $K$ by an isogeny of prime degree $\ell$, then so are $E_{1}^{\chi}$ and $E_{2}^{\chi}$ for every $\chi$, and the induced $\operatorname{maps} \operatorname{Sel}_{n}\left(E_{1}^{\chi} / K\right) \rightarrow \operatorname{Sel}_{n}\left(E_{2}^{\chi} / K\right)$ are isomorphisms for every $n$ prime to $\ell$. Thus in this case $E_{1}$ and $E_{2}$ are $n$-Selmer companions for every $n$ prime to $\ell$. For this reason we are mainly interested in nonisogenous $n$-Selmer companions, although the case of isogenies of degree dividing $n$ is still interesting.

Our main result is Theorem 3.1 below, which gives sufficient conditions for a pair of curves to be $p^{k}$-Selmer companions for a prime power $p^{k}$.

For example, we will show (see 4 (4) that the following pairs of non-isogenous curves, given with their labels from [2], are $p^{k}$-Selmer companions for $p^{k}$ as listed, over every number field $K$ (and in particular over $\mathbf{Q}$ ):

\begin{tabular}{|c|c|l|}
\hline$p^{k}$ & label & curve \\
\hline \hline \multirow{2}{*}{4} & $1242 L 1$ & $y^{2}+x y+y=x^{3}-x^{2}+x+1$ \\
& $1242 K 1$ & $y^{2}+x y+y=x^{3}-x^{2}-1666739-2448131309$ \\
\hline \hline \multirow{2}{*}{5} & $676 B 1$ & $y^{2}=x^{3}+x^{2}-4 x-12$ \\
& $676 E 1$ & $y^{2}=x^{3}-28561 x+1856465$ \\
\hline \hline \multirow{2}{*}{7} & $1026 N 1$ & $y^{2}+x y+y=x^{3}-x^{2}-34601 x-2468631$ \\
& $1026 O 1$ & $y^{2}+x y+y=x^{3}-x^{2}-4241 x+107353$ \\
\hline \hline \multirow{2}{*}{9} & $6555 D 1$ & $y^{2}+y=x^{3}-x^{2}+59335 x+3888371$ \\
& $6555 E 1$ & $y^{2}+y=x^{3}-x^{2}-33735977475 x-2384987222304844$ \\
\hline
\end{tabular}

A natural open problem is to give necessary and sufficient conditions for $p$ companionship, with a prime $p$. We have not found any example of a pair of $p$-Selmer companions having different conductors, or having different Galois action on their $p$-torsion points. In $\$ 7$ we discuss some related questions, including partial converses to Theorem 3.1 .

It also seems natural to expect that a given elliptic curve can have only finitely many $p$-Selmer companions, even if we allow the prime $p$ to vary. It is not difficult to show that for a given $E_{1} / K$ and fixed $p$, there are only finitely many $E_{2} / K$ satisfying the hypotheses of Theorem 3.1 because all such curves must have the same set of primes of bad reduction. See Proposition 7.1.

Much of what we do in this paper applies more generally to the Bloch-Kato Selmer groups [1] attached to a motive. The subtlest problem in the general case, as in the case of elliptic curves, is to understand the local condition at $p$ in the definition of the Bloch-Kato $p^{k}$-Selmer group, and how it changes under twists. It 
would be of interest to try to express that local condition only in terms of finite congruence information related to the motive.

Finally, it would be tempting to ask for rank companions, i.e., pairs of elliptic curves $E_{1}$ and $E_{2}$ over $K$ such that $\operatorname{rank}\left(E_{1}^{\chi}(K)\right)=\operatorname{rank}\left(E_{2}^{\chi}(K)\right)$ for every quadratic character of $K$. Equivalently, $E_{1}$ and $E_{2}$ are rank companions if $\operatorname{rank}\left(E_{1}(F)\right)=\operatorname{rank}\left(E_{2}(F)\right)$ for every quadratic extension $F$ of $K$. We expect that there are no non-isogenous rank companions; this is a stronger version of a question asked by Parshin and Zarhin [18, p. 94].

Notation. If $K$ is a field, we let $\bar{K}$ denote a fixed algebraic closure of $K$, and $G_{K}=$ $\operatorname{Gal}(\bar{K} / K)$. Let $\mathcal{X}(K):=\operatorname{Hom}\left(G_{K},\{ \pm 1\}\right)$ be the group of quadratic characters of $K$.

If $E$ is an elliptic curve over $K$, then $j(E)$ will denote the $j$-invariant of $E$ and $\Delta_{E}$ the discriminant of some model of $E$. If $m$ is a positive integer, then $E[m]$ will denote the kernel of multiplication by $m$ on $E$.

If $K$ is a local field and $E$ is an elliptic curve over $K$, then we will say that $E$ has potentially good reduction if $E$ has good reduction over some finite extension $F$ of $K$, including the case where $E$ has good reduction over $K$, i.e., $F=K$. Similarly we will say that $E$ has potentially multiplicative reduction if $E$ has multiplicative reduction over a finite extension $F$ of $K$, including the case where $E$ has multiplicative reduction over $K$.

\section{ElLiptic CURVES OVER LOCAL FIELDS}

For this section suppose that $K$ is a finite extension of $\mathbf{Q}_{\ell}$ for some rational prime $\ell$, and $E$ is an elliptic curve over $K$ with potentially multiplicative reduction. Let $\operatorname{ord}_{K}: K^{\times} \rightarrow \mathbf{Z}$ denote the valuation on $K$.

Definition 2.1. There is a unique character $\psi=\psi_{E / K} \in \mathcal{X}(K)$ such that the twist $E^{\psi}$ has split multiplicative reduction (see for example [23, §1.12]). We will call $\psi$ the splitting character of $E / K$.

The next proposition is due to Tate [30, Theorem 1].

Proposition 2.2 (Tate [30]). There is a Tate parameter $q=q_{E} \in K^{\times}$with $\operatorname{ord}_{K}(q)<0$, and an isomorphism

$$
\tau_{E / K}: \bar{K}^{\times} / q^{\mathbf{Z}} \stackrel{\sim}{\longrightarrow} E(\bar{K}) .
$$

If $E$ has split multiplicative reduction, then $\tau_{E / K}$ is $G_{K}$-equivariant. In general, we have

$$
\tau_{E / K}\left(u^{\sigma}\right)=\psi(\sigma) \tau_{E / K}(u)^{\sigma}
$$

for every $\sigma \in G_{K}$, where $\psi$ is the splitting character of $E / K$.

Let $\boldsymbol{\mu}_{m}$ denote the group of $m$-th roots of unity in $\bar{K}$, and

$$
\epsilon_{m}: G_{K} \rightarrow \operatorname{Aut}\left(\boldsymbol{\mu}_{m}\right) \stackrel{\sim}{\rightarrow}(\mathbf{Z} / m \mathbf{Z})^{\times}
$$

the $\bmod m$ cyclotomic character.

Definition 2.3. For $m>0$, define the canonical subgroup

$$
\mathcal{C}_{E / K}[m]:=\tau_{E / K}\left(\boldsymbol{\mu}_{m}\right) \subset E[m] .
$$

Lemma 2.4. There is a $G_{K}$-isomorphism $\mathcal{C}_{E / K}[m] \cong \boldsymbol{\mu}_{m} \otimes \psi_{E / K}$, i.e., $\mathcal{C}_{E / K}[m]$ is a cyclic group of order $m$ on which $G_{K}$ acts by the character $\epsilon_{m} \psi_{E / K}$. 
Proof. This is immediate from Proposition 2.2

Lemma 2.5. Suppose $E_{1}, E_{2}$ are elliptic curves over $K$ with potentially multiplicative reduction, $p$ is a prime, and $p \nmid \operatorname{ord}_{K}\left(j\left(E_{1}\right)\right)$. Suppose further that either $p \neq 2$, or $E_{1}$ and $E_{2}$ have multiplicative reduction.

Then for every $k \geq 1$ and every $G_{K}$-isomorphism $\lambda: E_{1}\left[p^{k}\right] \stackrel{\sim}{\rightarrow} E_{2}\left[p^{k}\right]$, we have

$$
\lambda\left(\mathcal{C}_{E_{1} / K}\left[p^{k}\right]\right)=\mathcal{C}_{E_{2} / K}\left[p^{k}\right] .
$$

Proof. Let $q_{i}$ be the Tate parameter of $E_{i} / K$, and $\psi_{i}$ the splitting character. Let $\tau_{i}=\tau_{E_{i} / K}$ be the map of Proposition 2.2

Fix a $G_{K}$-isomorphism $\lambda: E_{1}\left[p^{k}\right] \stackrel{\sim}{\rightarrow} E_{2}\left[p^{k}\right]$. By Proposition [2.2, for every $\sigma \in G_{K}$ and $\beta \in\left(\bar{K}^{\times} / q_{i}^{\mathbf{Z}}\right)\left[p^{k}\right]$, we have

$$
\psi_{i}(\sigma) \tau_{i}(\beta)^{\sigma}-\tau_{i}(\beta)=\tau_{i}\left(\beta^{\sigma} / \beta\right) \in \tau_{i}\left(\boldsymbol{\mu}_{p^{k}}\right)=\mathcal{C}_{E_{i} / K}\left[p^{k}\right] .
$$

Define

$$
B_{i}=\left\{z^{\sigma}-z: z \in E_{i}\left[p^{k}\right], \sigma \in G_{K}, \text { and } \psi_{1}(\sigma)=\psi_{2}(\sigma)=1\right\} .
$$

Then by (11) we have $B_{i} \subset \mathcal{C}_{E_{i} / K}\left[p^{k}\right]$, and since $\lambda$ is $G_{K}$-equivariant we have $\lambda\left(B_{1}\right)=B_{2}$.

Choose $\gamma \in \bar{K}$ with $\gamma^{p}=q_{1}$. We have $\operatorname{ord}_{K}\left(q_{1}\right)=-\operatorname{ord}_{K}\left(j\left(E_{1}\right)\right)$, so $\operatorname{ord}_{K}\left(q_{1}\right)$ is prime to $p$ and $[K(\gamma): K]=p$. We will show that there is a $\sigma \in G_{K}$ such that $\gamma^{\sigma} \neq \gamma$ and $\psi_{1}(\sigma)=\psi_{2}(\sigma)=1$.

Case 1: $E_{1}$ and $E_{2}$ have multiplicative reduction. Since $\operatorname{ord}_{K}\left(q_{1}\right)$ is prime to $p$, $K(\gamma) / K$ is ramified, so there is a $\sigma$ in the inertia group $I_{K} \subset G_{K}$ such that $\gamma^{\sigma} \neq \gamma$. If $E_{1}$ and $E_{2}$ have multiplicative reduction, then $\psi_{1}$ and $\psi_{2}$ are unramified, so $\psi_{1}(\sigma)=\psi_{2}(\sigma)=1$.

Case 2: $p>2$. Since $[K(\gamma): K]=p$, we have $\gamma \notin K\left(\boldsymbol{\mu}_{p}\right)$. Thus we can choose $\sigma_{0} \in G_{K\left(\boldsymbol{\mu}_{p}\right)}$ such that $\gamma^{\sigma_{0}} \neq \gamma$. Let $\sigma=\sigma_{0}^{2}$. Since $p \neq 2$ and $\sigma_{0}$ fixes $\gamma^{\sigma_{0}} / \gamma \in \boldsymbol{\mu}_{p}$, we have that $\gamma^{\sigma} / \gamma=\left(\gamma^{\sigma_{0}} / \gamma\right)^{2} \neq 1$. Since $\psi_{1}$ and $\psi_{2}$ are quadratic characters, we also have $\psi_{1}(\sigma)=\psi_{2}(\sigma)=1$.

Now choose $\beta \in \bar{K}$ with $\beta^{p^{k}}=q_{1}$. Then $\beta^{\sigma} / \beta$ is a primitive $p^{k}$-th root of unity, so $\tau_{1}\left(\beta^{\sigma} / \beta\right)$ generates $\mathcal{C}_{E_{1} / K}\left[p^{k}\right]$, and by (10) we have that $\tau_{1}\left(\beta^{\sigma} / \beta\right) \in B_{1}$. Therefore $B_{1}=\mathcal{C}_{E_{1} / K}\left[p^{k}\right]$, so

$$
\lambda\left(\mathcal{C}_{E_{1} / K}\left[p^{k}\right]\right)=\lambda\left(B_{1}\right)=B_{2} \subset \mathcal{C}_{E_{2} / K}\left[p^{k}\right] .
$$

The final inclusion must be an equality because both groups have order $p^{k}$ by Lemma 2.4.

\section{MAIN THEOREM}

Suppose for this section that $K$ is a number field. Fix a prime $p$ and a power $p^{k}$ of $p, k \geq 1$. Our main result is the following.

Theorem 3.1. Suppose $E_{1}$ and $E_{2}$ are elliptic curves over $K$. Let $S_{i}$ be the set of primes of $K$ where $E_{i}$ has potentially multiplicative reduction. Let $m=p^{k+1}$ if $p \leq 3$, and $m=p^{k}$ if $p>3$. Suppose further that:

(i) there is a $G_{K}$-isomorphism $E_{1}[m] \cong E_{2}[m]$,

(ii) $S_{1}=S_{2}$,

(iii) for all $\mathfrak{l} \in S_{1}=S_{2}$, the isomorphism of (i) sends $\mathcal{C}_{E_{1} / K_{\mathfrak{l}}}[m]$ to $\mathcal{C}_{E_{2} / K_{\mathfrak{l}}}[m]$, 
(iv) for every $\mathfrak{p}$ of $K$ above $p$, either

- $\mathfrak{p} \in S_{1}=S_{2}$, or

- $k=1, E_{1}$ and $E_{2}$ have good reduction at $\mathfrak{p}$, and the ramification degree $e(\mathfrak{p} / p)$ is less than $p-1$.

Then for every finite extension $F$ of $K$, and every $\chi \in \mathcal{X}(F)$, there is a canonical isomorphism

$$
\operatorname{Sel}_{p^{k}}\left(E_{1}^{\chi} / F\right) \cong \operatorname{Sel}_{p^{k}}\left(E_{2}^{\chi} / F\right) .
$$

In particular $E_{1}$ and $E_{2}$ are $p^{k}$-Selmer companions over every number field containing $K$.

A slightly stronger version of Theorem 3.1 will be proved in $\$ 6$ below. We first give some remarks, consequences and examples.

Remark 3.2. The proof of Theorem 3.1 will show that $\operatorname{Sel}_{p^{k}}\left(E_{1}^{\chi} / F\right), \operatorname{Sel}_{p^{k}}\left(E_{2}^{\chi} / F\right)$ are actually equal inside $H^{1}\left(F, E_{1}^{\chi}\left[p^{k}\right]\right)=H^{1}\left(F, E_{2}^{\chi}\left[p^{k}\right]\right)$, where we use the isomorphism $E_{1}\left[p^{k}\right] \cong E_{2}\left[p^{k}\right]$ to identify $H^{1}\left(F, E_{1}^{\chi}\left[p^{k}\right]\right)$ with $H^{1}\left(F, E_{2}^{\chi}\left[p^{k}\right]\right)$. See Definition 6.2 and the remarks following it.

Remark 3.3. A careful reading of the proof will show that when $p=3$, if $E_{1}$ and $E_{2}$ have no primes of additive reduction of Kodaira type II, IV, II* , or IV*, then we can take $m=3^{k}$ instead of $3^{k+1}$ in the hypotheses of Theorem 3.1. See Remark 5.2. In particular if $E$ is semistable, we can take $m=3^{k}$ instead of $m=3^{k+1}$.

Corollary 3.4. Suppose $K^{\prime}$ is a number field with a unique prime $\mathfrak{p}$ above $p$. Suppose $E_{1}$ and $E_{2}$ are elliptic curves over $K^{\prime}$ with potentially multiplicative reduction at $\mathfrak{p}$, and with potentially good reduction at all primes different from $\mathfrak{p}$. Let $m=p^{k+1}$ if $p \leq 3$, and $m=p^{k}$ if $p>3$. Then $E_{1}$ and $E_{2}$ are $p^{k}$-Selmer companions over every number field $K$ containing $K^{\prime}\left(E_{1}[m], E_{2}[m]\right)$.

Proof. Fix any group isomorphism $\lambda: E_{1}[m] \rightarrow E_{2}[m]$ that takes $\mathcal{C}_{E_{1} / K_{\mathfrak{p}}^{\prime}}[m]$ to $\mathcal{C}_{E_{2} / K_{\mathfrak{p}}^{\prime}}[m]$. If $K$ is a field containing $K^{\prime}\left(E_{1}[m], E_{2}[m]\right)$, then $\lambda$ is $G_{K^{-}}$-equivariant because $G_{K}$ acts trivially on both sides. We have $S_{1}=S_{2}$ is the set of all primes of $K$ above $\mathfrak{p}$, so all hypotheses of Theorem 3.1 are satisfied.

Remark 3.5. One can also twist elliptic curves by characters of order $\ell>2$. In this case the twist $E^{\chi}$ is an abelian variety of dimension $\ell-1$, with an action of the group of $\ell$-th roots of unity. One can study the $\mathfrak{n}$-Selmer groups $\operatorname{Sel}_{\mathfrak{n}}\left(E^{\chi} / K\right)$, where $\mathfrak{n}$ is an ideal of the cyclotomic field of $\ell$-th roots of unity, and ask when two elliptic curves $E_{1}, E_{2}$ have the property that $\operatorname{Sel}_{\mathfrak{n}}\left(E_{1}^{\chi} / K\right) \cong \operatorname{Sel}_{\mathfrak{n}}\left(E_{2}^{\chi} / K\right)$ for every character $\chi$ of $G_{K}$ of order $\ell$. An analogue of Theorem 3.1 holds in this case, with a similar proof.

\section{EXAmples}

Suppose we are given elliptic curves $E_{1}, E_{2}$ over $K$. We next introduce some tools to prove in practice that $E_{1}$ and $E_{2}$ satisfy the hypotheses of Theorem 3.1. Let $m=p^{k}$ or $p^{k+1}$ as in Theorem 3.1.

Remark 4.1. The most difficult hypothesis of Theorem 3.1 to check is (i), the existence of a $G_{K}$-isomorphism $E_{1}[m] \cong E_{2}[m]$. When $m \leq 5$ this can be done by computing explicitly the (genus zero) family of all elliptic curves $E$ over $K$ with $E[m] \cong E_{1}[m]$, and checking whether $E_{2}$ belongs to this family. 
For larger $m$, checking whether two given elliptic curves have isomorphic $m$ torsion subgroups is more subtle. A necessary condition for the existence of such an isomorphism is that for every prime $\mathfrak{l}$ of $K$ where $E_{1}$ and $E_{2}$ have good reduction, the traces of Frobenius Frobr acting on $E_{1}[\mathrm{~m}]$ and $E_{2}[\mathrm{~m}]$ are the same. In some cases this necessary condition can be turned into a sufficient condition (see for example [13]). We will take a more brute force approach, and for candidate curves that satisfy the necessary condition on traces for many primes, we will simply construct an isomorphism directly.

We will discuss both of these approaches in more detail in Appendix A.

It is natural to ask how common are pairs of non-isogenous curves $E_{1}, E_{2}$ with $E_{1}[m] \cong E_{2}[m]$ as $G_{K}$-modules. For some history of this question, see [8, 13, 15$]$. Along these lines, we raise the following question.

Question 4.2. Is there an integer $N$ (depending only on $K$, or perhaps only on the degree $[K: \mathbf{Q}])$ such that for every $m>N$, there are no pairs of non-isogenous elliptic curves $E_{1}, E_{2}$ over $K$ such that $E_{1}[m]$ is $G_{K}$-isomorphic to $E_{2}[m]$ ?

The reason one might imagine that the answer to Question 4.2 could be "yes" is that the Humbert surfaces $H(m)$ of level $m$, whose non-cuspidal points classify pairs of elliptic curves $\left(E, E^{\prime}\right)$ together with a symplectic isomorphism $E[m] \cong E^{\prime}[m]$, are of general type if $m \geq 13$. See [8]; the surfaces $H(m)$ are among the modular diagonal quotient surfaces treated there. Let $H(m)(K)^{\prime}$ denote the $K$-rational points of $H(m)$ associated to pairs $\left(E, E^{\prime}\right)$ of non-isogenous elliptic curves. It has long been conjectured (see the discussion in loc. cit.) that for any number field $K$ the sets $H(m)(K)^{\prime}$ are finite for sufficiently large $m$. Question 4.2 asks whether for $m$ sufficiently large (depending only on $K$, or on $[K: \mathbf{Q}]$ ) the sets $H(m)(K)^{\prime}$ are empty.

Remark 4.3. It is well known (see for example [29]) that an elliptic curve $E$ has potentially multiplicative reduction at a prime $\mathfrak{l}$ if and only if $\operatorname{ord}_{\mathfrak{l}}(j(E))<0$. Thus $S_{1}=S_{2}$ if and only if $j\left(E_{1}\right)$ and $j\left(E_{2}\right)$ have the same primes in their denominators. This makes it very easy to check hypothesis (ii) of Theorem 3.1 .

Hypothesis (iii) can often be verified by using Lemma 2.5.

Example 4.4. Consider the first example of the Introduction, with $K=\mathbf{Q}, p^{k}=4$, and

$$
\begin{aligned}
& E_{1}: y^{2}+x y+y=x^{3}-x^{2}+x+1 \\
& E_{2}: y^{2}+x y+y=x^{3}-x^{2}-1666739-2448131309 .
\end{aligned}
$$

These are the curves $1242 \mathrm{~L} 1$ and $1242 \mathrm{~K} 1$, respectively, in [2. The following proposition can be proved using the method of $\$$ A.2 of Appendix $\mathrm{A}$, using computations in Sage [22] and PARI/GP [17]. Its proof will be sketched at the end of 4.2 .

Proposition 4.5. There is a $G_{\mathbf{Q}}$-isomorphism $E_{1}[8] \cong E_{2}[8]$.

Computing the $j$-invariants gives

$$
j\left(E_{1}\right)=\frac{3^{3} \cdot 7^{3}}{2 \cdot 23}, \quad j\left(E_{2}\right)=\frac{3 \cdot 987697^{3}}{2^{49} \cdot 23}
$$

so $S_{1}=S_{2}=\{2,23\}$. Combined with Proposition 4.5 this shows that Theorem 3.1(i, ii, iv) are satisfied. For $\ell \in\{2,23\}$ both $E_{1}$ and $E_{2}$ have multiplicative 
reduction at $\ell$, and $\operatorname{ord}_{\ell}\left(j\left(E_{1}\right)\right)$ is odd, so Lemma 2.5 shows that Theorem 3.1](iii) is satisfied.

Thus all hypotheses of Theorem 3.1 are satisfied, and we conclude that $E_{1}$ and $E_{2}$ are 4-Selmer companions over every number field.

The third and fourth examples of the Introduction, with $p^{k}=7$ and 9 , are proved in exactly the same way, using $₫$ A.2 to construct a $G_{\mathbf{Q}}$-isomorphism $E_{1}[m] \cong E_{2}[m]$. For the $p^{k}=9$ example, we use Remark 3.3 so that (since $E_{1}$ and $E_{2}$ are semistable) we can take $m=9$ in Theorem 3.1 instead of $m=27$.

Example 4.6. Consider the second example of the Introduction, with $K=\mathbf{Q}$, $p^{k}=5$, and

$$
\begin{aligned}
& E_{1}: y^{2}=x^{3}+x^{2}-4 x-12, \\
& E_{2}: y^{2}=x^{3}-28561 x+1856465 .
\end{aligned}
$$

These are the curves $676 B 1$ and $676 E 1$, respectively, in [2]. We will prove the following proposition in A.1 of Appendix A

Proposition 4.7. There is a $G_{\mathbf{Q}^{-}}$-isomorphism $E_{1}[5] \cong E_{2}[5]$.

Both $E_{1}$ and $E_{2}$ have conductor $676=2^{2} \cdot 13^{2}, j\left(E_{1}\right)=-208$, and $j\left(E_{2}\right)=$ 1168128. Thus $E_{1}, E_{2}$ have good reduction at 5 and potentially good reduction everywhere, so $S_{1}=S_{2}$ is the empty set and all hypotheses of Theorem 3.1 are satisfied. We conclude that $E_{1}$ and $E_{2}$ are 5 -Selmer companions over every number field.

The strategy of Example 4.6, along with the method described in $\$$ A.1 of Appendix A, works in exactly the same way to give many more examples of $p$-Selmer companions over $\mathbf{Q}$ with $p=2,3$ or 5 .

\section{Elliptic CURVES OVER LOCAL FIELdS, CONTINUED}

For this section let $K$ be a finite extension of $\mathbf{Q}_{\ell}$ or of $\mathbf{R}$. Fix a rational prime power $p^{k}$. For every elliptic curve $E / K$, let $\kappa_{E}=\kappa_{E / K}$ denote the Kummer map

$$
\kappa_{E / K}: E(K) \longrightarrow H^{1}\left(K, E\left[p^{k}\right]\right) .
$$

For the proof of Theorem 3.1 we need to understand the image of $\kappa_{E}$, because the Selmer group $\operatorname{Sel}_{p^{k}}(E / F)$ (see Definition 6.2 below) depends on the $G_{F}$-module $E\left[p^{k}\right]$ and the images of the maps (3) as $K$ runs through all the completions of the number field $F$. We will consider several cases.

\subsection{Potentially good reduction.}

Lemma 5.1. Suppose that either $K$ is archimedean, or $K$ is non-archimedean of residue characteristic different from $p$ and $E$ has bad, potentially good reduction.

(i) If $p \leq 3$, then image $\left(\kappa_{E}\right)$ is represented by the cocycles

$$
\left\{c_{t}: t \in E\left[p^{k+1}\right], p^{k} t \in E(K)\right\},
$$

where $c_{t}$ is defined by $c_{t}(\sigma)=t^{\sigma}-t$ for $\sigma \in G_{K}$.

(ii) If $p>3$, or if $v$ is archimedean and $p>2$, then image $\left(\kappa_{E}\right)=0$. 
Proof. We consider the archimedean and non-archimedean cases separately.

Case 1: $K$ is archimedean. In this case (ii) is clear, because $G_{K}$ has order 1 or 2 so $H^{1}\left(K, E\left[p^{k}\right]\right)=0$.

For every $p$, the map $E(K)[p] \rightarrow E(K) / p^{k} E(K)$ is surjective, so image $\left(\kappa_{E}\right)=$ $\kappa_{E}(E(K)[p])$. If $s \in E(K)[p]$, then $\kappa_{E}(s)$ is represented by the cocycle $c_{t}$ for (every) $t \in E\left[p^{k+1}\right]$ with $p^{k} t=s$. This proves (i) in this case.

Case 2: $K$ is non-archimedean of residue characteristic different from $p$ and $E$ has bad, potentially good reduction. Consider the filtration

$$
E_{1}(K) \subset E_{0}(K) \subset E(K)
$$

where $E_{0}(K)$ is the subgroup of points with non-singular reduction, and $E_{1}(K)$ is the subgroup of points that reduce to zero [27. Chapter VII]. Then $E_{1}(K)$ is a pro- $\ell$-group, where $\ell$ is the residue characteristic of $K$. Since $E$ has additive reduction, $E_{0}(K) / E_{1}(K)$ is isomorphic to the additive group of the residue field of $K$, also an $\ell$-group. The description of $E(K) / E_{0}(K)$ in [29] shows that the $p$-part of $E(K) / E_{0}(K)$ is killed by $p$, and is trivial if $p>3$. Hence $E(K) / p^{k} E(K)=0$ if $p>$ 3 , and $E(K)[p] \rightarrow E(K) / p^{k} E(K)$ is surjective for every $p$. Therefore image $\left(\kappa_{E}\right)=0$ if $p>3$, and (as in Case 1) image $\left(\kappa_{E}\right)=\kappa_{E}(E(K)[p])$ is represented by the cocycles $\left\{c_{t}: t \in E\left[p^{k+1}\right], p^{k} t \in E(K)\right\}$ if $p \leq 3$. This completes the proof.

Remark 5.2. Suppose $p=3$. If $K$ is non-archimedean and the Kodaira type of the reduction of $E$ is not II, IV, $\mathrm{II}^{*}$, or $\mathrm{IV}^{*}$, then [29] shows that $E(K) / E_{0}(K)$ has order prime to 3 . Thus in those cases we have image $\left(\kappa_{E}\right)=0$, just as when $p>3$. This allows us to take $m=3^{k}$ instead of $m=3^{k+1}$ in Theorem 3.1 in this case (see Remark 3.3).

Lemma 5.3. Suppose $K$ is non-archimedean of residue characteristic not dividing $m$, and $E_{1}, E_{2}$ are elliptic curves over $K$ with potentially good reduction. If $m \geq 3$ and $E_{1}[m] \cong E_{2}[m]$ as $G_{K}$-modules, then either both $E_{1}$ and $E_{2}$ have good reduction, or both have bad reduction.

Proof. This follows directly from [24, Corollary 2(b) of Theorem 2].

5.2. Potentially multiplicative reduction. Recall that if $E$ has potentially multiplicative reduction, then Proposition 2.2 gives a parameter $q$ and an isomorphism $\tau_{E / K}: \bar{K}^{\times} / q^{\mathbf{Z}} \rightarrow E(\bar{K})$.

Lemma 5.4. Suppose $E / K$ has split multiplicative reduction. Then image $\left(\kappa_{E}\right)$ is the image of the composition

$$
K^{\times} \longrightarrow H^{1}\left(K, \boldsymbol{\mu}_{p^{k}}\right) \longrightarrow H^{1}\left(K, E\left[p^{k}\right]\right)
$$

where the first map is the classical Kummer map, and the second is induced by the inclusion $\tau_{E / K}: \boldsymbol{\mu}_{p^{k}} \hookrightarrow E\left[p^{k}\right]$.

Proof. This follows directly from the commutativity of the diagram

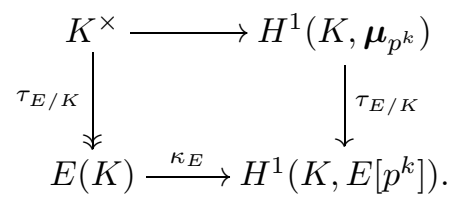


Definition 5.5. Suppose $L$ is a quadratic extension of $K$. Let $N_{L / K}: L^{\times} \rightarrow K^{\times}$ be the norm map, and let $\psi \in \mathcal{X}(K)$ be the quadratic character attached to $L / K$. Let $\boldsymbol{\mu}_{p^{k}} \otimes \psi$ denote a copy of $\boldsymbol{\mu}_{p^{k}}$ with $G_{K}$ acting by $\epsilon_{p^{k}} \psi$ instead of $\epsilon_{p^{k}}$, and define a twisted Kummer map

$$
\nu_{L / K}: \operatorname{ker}\left(N_{L / K}\right) \longrightarrow H^{1}\left(K, \boldsymbol{\mu}_{p^{k}} \otimes \psi\right)
$$

where for $x \in \operatorname{ker}\left(N_{L / K}\right)$, we choose $u \in \bar{K}$ such that $u^{p^{k}}=x$, and then $\nu_{L / K}(x)$ is represented by the cocycle $\sigma \mapsto\left(u^{\sigma}\right)^{\psi(\sigma)} / u$. We leave it as an exercise to show that $\sigma \mapsto\left(u^{\sigma}\right)^{\psi(\sigma)} / u$ is indeed a cocycle with values in $\boldsymbol{\mu}_{p^{k}} \otimes \psi$, when $x \in \operatorname{ker}\left(N_{L / K}\right)$.

Lemma 5.6. Suppose $E / K$ has potentially multiplicative reduction, with nontrivial splitting character $\psi$ (i.e., $E / K$ does not have split multiplicative reduction). Let $q \in K^{\times}$be the Tate parameter, and let $L$ be the quadratic extension of $K$ attached to $\psi$. Let $\mathcal{H}$ be the image of the composition

$$
\operatorname{ker}\left(N_{L / K}\right) \stackrel{\nu_{L / K}}{\longrightarrow} H^{1}\left(K, \boldsymbol{\mu}_{p^{k}} \otimes \psi\right) \stackrel{\tau_{E / K}}{\longrightarrow} H^{1}\left(K, E\left[p^{k}\right]\right) .
$$

(i) If $p>2$, or if $p=2$ and $q \notin N_{L / K} L^{\times}$, then image $\left(\kappa_{E}\right)=\mathcal{H}$.

(ii) Suppose $p=2$ and $q=N_{L / K}(\beta)$ with $\beta \in L^{\times}$. Then image $\left(\kappa_{E}\right)$ is the subgroup of $H^{1}\left(K, E\left[p^{k}\right]\right)$ generated by $\mathcal{H}$ and the class of the cocycle

$$
\sigma \mapsto \tau_{E / K}\left(\left(\alpha^{\sigma}\right)^{\psi(\sigma)} / \alpha\right)
$$

where $\alpha \in \bar{K}^{\times}$and $\alpha^{2^{k}}=\beta$.

Proof. Consider the commutative diagram with exact rows

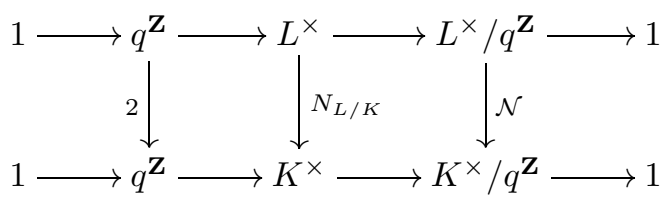

where we denote by $\mathcal{N}$ the right-hand norm map. The snake lemma shows that the map $\operatorname{ker}\left(N_{L / K}\right) \rightarrow \operatorname{ker}(\mathcal{N})$ is surjective if $q \notin N_{L / K} L^{\times}$, and if $q=N_{L / K}(\beta)$, then the cokernel of that map has order 2 , and $\operatorname{ker}(\mathcal{N})$ is generated by the image of $\operatorname{ker}\left(N_{L / K}\right)$ and $\beta$.

Proposition 2.2 shows that we have a commutative diagram

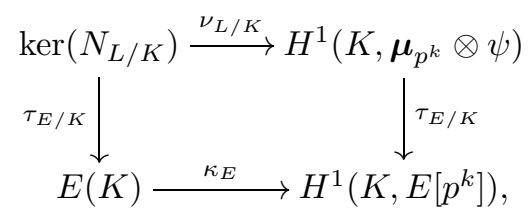

and $\tau_{E / K}: \operatorname{ker}(\mathcal{N}) \rightarrow E(K)$ is an isomorphism. (To see this last fact, note that $\tau_{E / K}: L^{\times} / q^{\mathbf{Z}} \rightarrow E(L)$ is a group isomorphism satisfying $\tau_{E / K}\left(u^{\sigma}\right)=-\tau_{E / K}(u)^{\sigma}$ if $\sigma$ is the non-trivial automorphism of $L / K$, so $\tau_{E / K}(u) \in E(K)$ if and only if $u^{\sigma}=u^{-1}$, i.e., if and only if $\mathcal{N}(u)=1$.) It follows that image $\left(\kappa_{E}\right)=\mathcal{H}$ if $p>2$ or if $q \notin N_{L / K} L^{\times}$, and if $q=N_{L / K}(\beta)$, then image $\left(\kappa_{E}\right)$ is generated by $\mathcal{H}$ and $\kappa_{E}\left(\tau_{E / K}(\beta)\right)$.

Suppose $p=2$ and $q=N_{L / K}(\beta)$, and choose $\alpha \in \bar{K}^{\times}$with $\alpha^{2^{k}}=\beta$. Then by definition $\kappa_{E}\left(\tau_{E / K}(\beta)\right)$ is represented by the cocycle $\sigma \mapsto \tau_{E / K}(\alpha)^{\sigma}-\tau_{E / K}(\alpha)$, 
and by Proposition 2.2

$$
\tau_{E / K}(\alpha)^{\sigma}-\tau_{E / K}(\alpha)=\psi(\sigma) \tau_{E / K}\left(\alpha^{\sigma}\right)-\tau_{E / K}(\alpha)=\tau_{E / K}\left(\left(\alpha^{\sigma}\right)^{\psi(\sigma)} / \alpha\right) .
$$

This completes the proof of the lemma.

Lemma 5.7. Let $m=2^{k+1}$ if $p=2$, and $m=p^{k}$ if $p>2$. Suppose $E_{1}$ and $E_{2}$ are elliptic curves over $K$ with potentially multiplicative reduction, and there is a $G_{K}$-isomorphism $E_{1}[m] \cong E_{2}[m]$ that identifies $\mathcal{C}_{E_{1} / K}[m]$ with $\mathcal{C}_{E_{2} / K}[m]$.

(i) The splitting characters of $E_{1}$ and $E_{2}$ are equal.

(ii) Suppose $p=2$. Let $q_{i}$ be the Tate parameter of $E_{i}$, and $\pi_{1} \in \bar{K}^{\times}$with $\pi_{1}^{2^{k}}=q_{1}$. Then there is an $x \in K^{\times}$and an odd integer $n$ such that $q_{2}^{n}=q_{1} x^{m}$ and $\tau_{E_{1} / K}\left(\pi_{1}\right)=\tau_{E_{2} / K}\left(\pi_{1} x^{2}\right)$ in $E_{1}\left[2^{k}\right]=E_{2}\left[2^{k}\right]$.

Proof. Since $\mathcal{C}_{E_{1} / K}[m]$ and $\mathcal{C}_{E_{1} / K}[m]$ are isomorphic $G_{K}$-modules, and $m>2$, assertion (i) follows directly from Lemma 2.4.

Suppose $p=2$, and let $\lambda: E_{1}[m] \rightarrow E_{2}[m]$ be the $G_{K}$-isomorphism. We will abbreviate $\tau_{i}:=\tau_{E_{i} / K}$. Replacing $\lambda$ by an odd multiple of $\lambda$ if necessary, we may assume that the diagram

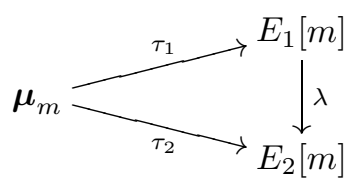

commutes.

Fix $\beta_{1} \in \bar{K}^{\times}$such that $\beta_{1}^{m}=q_{1}$. Then $\tau_{1}\left(\beta_{1}\right) \in E_{1}[m]$, and we fix $\beta_{2} \in \bar{K}^{\times}$such that $\tau_{2}\left(\beta_{2}\right)=\lambda\left(\tau_{1}\left(\beta_{1}\right)\right)$. Since $\tau_{2}\left(\beta_{2}\right) \in E_{2}[m]$, we have $\beta_{2}^{m} \in q_{2}^{\mathbf{Z}}$, say $\beta_{2}^{m}=q_{2}^{n}$. If $n$ is even, then $\beta_{2}^{m / 2}= \pm q_{2}^{n / 2}$, so $\tau_{2}\left(\beta_{2}^{m / 2}\right)=\tau_{2}( \pm 1) \in \mathcal{C}_{E_{2} / K}[m]$. But $\tau_{1}\left(\beta_{1}^{m / 2}\right) \notin$ $\mathcal{C}_{E_{1} / K}[m]$, so this is impossible and $n$ must be odd.

Let $\psi$ denote the common splitting character of $E_{1}$ and $E_{2}$. By Proposition 2.2 . for every $\sigma \in G_{K}$ we have

$$
\tau_{i}\left(\beta_{i}^{\sigma} / \beta_{i}\right)=\tau_{i}\left(\beta_{i}^{\sigma}\right)-\tau_{i}\left(\beta_{i}\right)=\psi(\sigma) \tau_{i}\left(\beta_{i}\right)^{\sigma}-\tau_{i}\left(\beta_{i}\right) .
$$

Applying $\lambda$ we see that $\lambda\left(\tau_{1}\left(\beta_{1}^{\sigma} / \beta_{1}\right)\right)=\tau_{2}\left(\beta_{2}^{\sigma} / \beta_{2}\right)$. But $\beta_{i}^{\sigma} / \beta_{i} \in \boldsymbol{\mu}_{m}$, so by (41) we have $\beta_{1}^{\sigma} / \beta_{1}=\beta_{2}^{\sigma} / \beta_{2}$. Thus $\left(\beta_{2} / \beta_{1}\right)^{\sigma}=\beta_{2} / \beta_{1}$ for every $\sigma \in G_{K}$, so $\beta_{2} / \beta_{1} \in K$.

Let $x=\beta_{2} / \beta_{1}$. Then $q_{2}^{n}=q_{1} x^{m}$, and if we take $\pi_{1}=\beta_{1}^{2}$, then $\pi_{1}^{2^{k}}=q_{1}$ and

$$
\tau_{2}\left(\pi_{1} x^{2}\right)=\tau_{2}\left(\pi_{1} \beta_{2}^{2} / \beta_{1}^{2}\right)=\tau_{2}\left(\beta_{2}^{2}\right)=\lambda\left(\tau_{1}\left(\beta_{1}^{2}\right)\right)=\lambda\left(\tau_{1}\left(\pi_{1}\right)\right) .
$$

This proves (ii).

5.3. The group scheme kernel of $p^{k}$. Let $\mathcal{O}$ be the ring of integers of $K$ and $\mathbf{F}$ the residue field. Let $\mathcal{E}$ be the Néron model of $E$, so $\mathcal{E}$ is a smooth group scheme over $\mathcal{O}$. Let $\mathcal{E}^{0}$ be its connected component at the identity, so $\mathcal{E}^{0}$ is an open subgroup scheme of $\mathcal{E}$. Let $\mathcal{E}_{/ \mathbf{F}}^{0} \subset \mathcal{E}_{/ \mathbf{F}}$ be the closed fibers of these group schemes, and $\Phi=\mathcal{E}_{/ \mathbf{F}} / \mathcal{E}_{/ \mathbf{F}}^{0}$ the group of components of the closed fiber, viewed as a (finite étale) group scheme over $\mathbf{F}$. Let $\mathcal{E}\left[p^{k}\right]$ be the kernel of multiplication by $p^{k}$ on $\mathcal{E}$; this is a group scheme over $\mathcal{O}$ with finite generic fiber. 
Proposition 5.8. Suppose that multiplication by $p$ induces a faithfully flat morphism $\mathcal{E} \rightarrow \mathcal{E}$ over $\mathcal{O}$. Then $\mathcal{E}\left[p^{k}\right]$ is a quasi-finite flat group scheme over $\mathcal{O}$, and image $\left(\kappa_{E}\right)$ is the image of the composition

$$
H_{\text {fppf }}^{1}\left(\operatorname{Spec}(\mathcal{O}), \mathcal{E}\left[p^{k}\right]\right) \longrightarrow H_{\text {fppf }}^{1}\left(\operatorname{Spec}(K), \mathcal{E}\left[p^{k}\right]\right) \stackrel{\sim}{\longrightarrow} H^{1}\left(K, E\left[p^{k}\right]\right)
$$

where $H_{\mathrm{fppf}}^{1}$ means cohomology of abelian group schemes, computed in the fppf topology.

Proof. This follows from [14, Lemma 5.1(iii)]. The long exact cohomology sequence attached to $0 \rightarrow \mathcal{E}\left[p^{k}\right] \rightarrow \mathcal{E} \stackrel{p^{k}}{\rightarrow} \mathcal{E} \rightarrow 0$ gives

$$
\begin{aligned}
\longrightarrow H_{\mathrm{fppf}}^{0}(\operatorname{Spec}(\mathcal{O}), \mathcal{E}) \stackrel{p^{k}}{\longrightarrow} & H_{\mathrm{fppf}}^{0}(\operatorname{Spec}(\mathcal{O}), \mathcal{E}) \\
\longrightarrow & H_{\mathrm{fppf}}^{1}\left(\operatorname{Spec}(\mathcal{O}), \mathcal{E}\left[p^{k}\right]\right) \longrightarrow H_{\mathrm{fppf}}^{1}(\operatorname{Spec}(\mathcal{O}), \mathcal{E})\left[p^{k}\right] .
\end{aligned}
$$

We have $H_{\text {fppf }}^{0}(\operatorname{Spec}(\mathcal{O}), \mathcal{E})=E(K)$, and $H_{\text {fppf }}^{1}(\operatorname{Spec}(\mathcal{O}), \mathcal{E})\left[p^{k}\right]=H^{1}(\mathbf{F}, \Phi)\left[p^{k}\right]$ by [14, Lemma 5.1(iii)]. It follows from our assumption on $p$ that $\Phi\left[p^{k}\right]=0$, so the upper left vertical map in the following commutative diagram is an isomorphism:

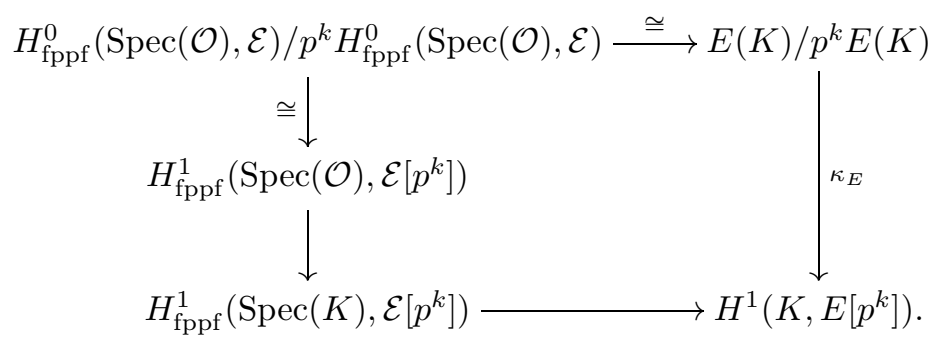

The proposition follows.

Remark 5.9. If $K$ has residue characteristic $p$, then multiplication by $p$ induces a faithfully flat endomorphism of $\mathcal{E}$ if and only if $E$ has good or multiplicative reduction and $p$ does not divide the order of $\Phi$.

\section{Proof of Theorem 3.1}

The following is a slightly stronger version of Theorem 3.1. We will prove Theorem 6.1 below, and then derive Theorem 3.1 from it. Fix a prime power $p^{k}$ and a number field $K$. Recall that for every field $F$, we let $\mathcal{X}(F)=\operatorname{Hom}\left(G_{F},\{ \pm 1\}\right)$.

Theorem 6.1. Suppose $E_{1}$ and $E_{2}$ are elliptic curves over $K$. Let $S_{i}$ be the set of primes of $K$ where $E_{i}$ has potentially multiplicative reduction. Let $m=p^{k+1}$ if $p \leq 3$, and $m=p^{k}$ if $p>3$. Suppose further that:

(i) there is a $G_{K}$-isomorphism $E_{1}[m] \cong E_{2}[m]$,

(ii) $S_{1}=S_{2}$,

(iii) for all $\mathfrak{l} \in S_{1}=S_{2}$, the isomorphism of (i) sends $\mathcal{C}_{E_{1} / K_{\mathfrak{l}}}[m]$ to $\mathcal{C}_{E_{2} / K_{\mathfrak{l}}}[m]$,

(iv) for every prime $v$ above $p$, either

- $E_{1}$ and $E_{2}$ have potentially multiplicative reduction at $v$, or

- $p>2, E_{1}$ and $E_{2}$ have good reduction at $v$, and the isomorphism of (i) extends to an isomorphism $\mathcal{E}_{1}\left[p^{k}\right] \cong \mathcal{E}_{2}\left[p^{k}\right]$ over $\operatorname{Spec}\left(\mathcal{O}_{K_{v}}\right)$, where the group scheme $\mathcal{E}_{i}\left[p^{k}\right]$ is the kernel of multiplication by $p^{k}$ in the Néron model of $E_{i}$. 
Then for every finite extension $F$ of $K$, and every $\chi \in \mathcal{X}(F)$, there is a canonical isomorphism

$$
\operatorname{Sel}_{p^{k}}\left(E_{1}^{\chi} / F\right) \cong \operatorname{Sel}_{p^{k}}\left(E_{2}^{\chi} / F\right) .
$$

In particular $E_{1}$ and $E_{2}$ are $p^{k}$-Selmer companions over every number field containing $K$.

Definition 6.2. The $p^{k}$-Selmer group $\operatorname{Sel}_{p^{k}}(E / K) \subset H^{1}\left(K, E\left[p^{k}\right]\right)$ of an elliptic curve $E$ over $K$ is

$$
\operatorname{Sel}_{p^{k}}(E / K):=\operatorname{ker}\left(H^{1}\left(K, E\left[p^{k}\right]\right) \longrightarrow \bigoplus_{v} H^{1}\left(K_{v}, E\left[p^{k}\right]\right) / \operatorname{image}\left(\kappa_{E / K_{v}}\right)\right) .
$$

A $G_{K}$-isomorphism $E_{1}\left[p^{k}\right] \cong E_{2}\left[p^{k}\right]$ allows us to identify

$$
H^{1}\left(K, E_{1}\left[p^{k}\right]\right)=H^{1}\left(K, E_{2}\left[p^{k}\right]\right), \quad H^{1}\left(K_{v}, E_{1}\left[p^{k}\right]\right)=H^{1}\left(K_{v}, E_{2}\left[p^{k}\right]\right) \text { for every } v .
$$

With these identifications, under the hypotheses of Theorems 3.1 and 6.1, we will show that the images of the horizontal Kummer maps

$$
\begin{aligned}
& E_{1}^{\chi}\left(K_{v}\right) \stackrel{\kappa_{E_{1}^{\chi} / K_{v}}}{\longrightarrow} H^{1}\left(K_{v}, E_{1}\left[p^{k}\right]\right) \\
& E_{2}^{\chi}\left(K_{v}\right) \stackrel{\kappa_{E_{2}^{\chi} / K_{v}}}{\longrightarrow} H^{1}\left(K_{v}, E_{2}\left[p^{k}\right]\right)
\end{aligned}
$$

are equal for every $v$ and for every $\chi \in \mathcal{X}\left(K_{v}\right)$. It will then follow from the definition that $\operatorname{Sel}_{p^{k}}\left(E_{1}^{\chi} / K\right)=\operatorname{Sel}_{p^{k}}\left(E_{2}^{\chi} / K\right)$ inside $H^{1}\left(K, E_{1}\left[p^{k}\right]\right)=H^{1}\left(K, E_{2}\left[p^{k}\right]\right)$ for every $\chi$. This gives the canonical isomorphisms referred to in Theorems 3.1 and 6.1

Proof of Theorem 6.1. Suppose $E_{1}$ and $E_{2}$ satisfy the hypotheses of Theorem 6.1. We will show that image $\left(\kappa_{E_{1}^{\chi} / K_{v}}\right)=\operatorname{image}\left(\kappa_{E_{2}^{\chi} / K_{v}}\right)$ for every place $v$ of $K$ and every $\chi \in \mathcal{X}(K)$. Note that except in the case where $v \mid p$ and $E_{1}, E_{2}$ have good reduction at $p$ (Case 5 below), if the pair $\left(E_{1}, E_{2}\right)$ satisfies the hypotheses of Theorem 6.1 then so does the pair $\left(E_{1}^{\chi}, E_{2}^{\chi}\right)$ for every $\chi \in \mathcal{X}(K)$. Thus in Cases $1-4$, it is enough to consider $\chi=1$. We will identify $E_{1}\left[p^{k}\right]$ with $E_{2}\left[p^{k}\right]$ using the given isomorphism, and denote this $G_{K}$-module simply by $E\left[p^{k}\right]$.

We split the proof into several cases.

Case 1: $v \mid \infty$. By Lemma 5.1, image $\left(\kappa_{E_{i} / K_{v}}\right)$ is zero if $p>2$, and is completely determined by the $G_{K}$-module $E_{i}\left[2^{k+1}\right]$ if $p=2$. But if $p=2$, then by assumption $E_{1}\left[2^{k+1}\right] \cong E_{2}\left[2^{k+1}\right]$ as $G_{K}$-modules, so image $\left(\kappa_{E_{1} / K_{v}}\right)=\operatorname{image}\left(\kappa_{E_{2} / K_{v}}\right)$.

Case 2: $v \nmid \infty$ and $E_{1}$ has bad, potentially good reduction at $v$. By our assumptions, $v \nmid p$ and $E_{2}$ also has potentially good reduction at $v$. By Lemma 5.3, the reduction of $E_{2}$ is also bad. By Lemma [5.1, we again see that image $\left(\kappa_{E_{i} / K_{v}}\right)$ is zero if $p>3$, and in general is completely determined by the $G_{K}$-module $E_{i}[m]$, so $\operatorname{image}\left(\kappa_{E_{1} / K_{v}}\right)=\operatorname{image}\left(\kappa_{E_{2} / K_{v}}\right)$.

Case 3: $v \nmid p \infty$ and $E_{1}$ has good reduction at $v$. By our assumptions, $E_{2}$ has potentially good reduction at $v$. By Lemma 5.3 the reduction of $E_{2}$ is also good. It follows that for $i=1,2$, the image of $\kappa_{E_{i} / K_{v}}$ is the unramified subgroup of $H^{1}\left(K_{v}, E\left[p^{k}\right]\right)$ (see [3, Lemma 4.1]), i.e.,

$$
\operatorname{image}\left(\kappa_{E_{i} / K_{v}}\right)=\operatorname{ker}\left(H^{1}\left(K_{v}, E\left[p^{k}\right]\right) \rightarrow H^{1}\left(K_{v}^{\mathrm{ur}}, E\left[p^{k}\right]\right)\right)
$$

where $K_{v}^{\text {ur }}$ is the maximal unramified extension of $K_{v}$. Since this subgroup is independent of $i$, we have image $\left(\kappa_{E_{1} / K_{v}}\right)=\operatorname{image}\left(\kappa_{E_{2} / K_{v}}\right)$. 
Case 4: $v \nmid \infty$ and $E_{1}$ has potentially multiplicative reduction at $v$. By our assumptions, $E_{2}$ also has potentially multiplicative reduction at $v$, and the images of $H^{1}\left(K_{v}, \boldsymbol{\mu}_{p^{k}}\right) \rightarrow H^{1}\left(K_{v}, E_{i}\left[p^{k}\right]\right)$ are identified by the isomorphism $E_{1}\left[p^{k}\right] \cong E_{2}\left[p^{k}\right]$.

Suppose first that $E_{1}$ has split multiplicative reduction. Then so does $E_{2}$ (by Lemma $5.7(\mathrm{i}))$, and then Lemma 5.4 shows that image $\left(\kappa_{E_{1} / K_{v}}\right)=\operatorname{image}\left(\kappa_{E_{2} / K_{v}}\right)$.

Now suppose $E_{1}$ does not have split multiplicative reduction, and let $L$ be the quadratic extension corresponding to the splitting character of $E_{1} / K_{v}$. By Lemma 5.7(i), the splitting characters of $E_{1}$ and $E_{2}$ are equal. Let $q_{i}$ be the Tate parameter of $E_{i}$. By Lemma 5.7(ii), if $p=2$ we have $q_{1} \in N_{L / K_{v}} L^{\times} \Longleftrightarrow q_{2} \in N_{L / K_{v}} L^{\times}$.

If $p>2$, or if $p=2$ and $q_{1}, q_{2} \notin N_{L / K_{v}} L^{\times}$, then it follows from Lemma 5.6(i) that image $\left(\kappa_{E_{1} / K_{v}}\right)=\operatorname{image}\left(\kappa_{E_{2} / K_{v}}\right)$.

Finally, suppose that $p=2$ and $q_{1} \in N_{L / K_{v}} L^{\times}$, say $q_{1}=N_{L / K_{v}}\left(\beta_{1}\right)$. Let $x \in K_{v}^{\times}$and the odd integer $n$ be as in Lemma 5.7(ii), so $q_{2}^{n}=q_{1} x^{m}$, and we set $\beta_{2}=\left(\beta_{1} x^{2^{k}}\right)^{n}$ so $N_{L / K_{v}}\left(\beta_{2}\right)=q_{2}^{n}$. Fix $\alpha_{1} \in \bar{K}_{v}^{\times}$with $\alpha_{1}^{2^{k}}=\beta_{1}$, and set $\alpha_{2}=\left(\alpha_{1} x\right)^{n}$, so $\alpha_{2}^{2^{k}}=\beta_{2}$. Using Lemma 5.6(ii), to show that image $\left(\kappa_{E_{1} / K_{v}}\right)=$ $\operatorname{image}\left(\kappa_{E_{2} / K_{v}}\right)$ it is enough to show that the two cocycles

$$
c_{i}: \sigma \mapsto \tau_{E_{i} / K_{v}}\left(\left(\alpha_{i}^{\sigma}\right)^{\psi(\sigma)} / \alpha_{i}\right)
$$

generate the same subgroup of $H^{1}\left(K_{v}, E\left[2^{k}\right]\right)$, for $i=1,2$.

If $\sigma$ fixes $L$ (i.e., $\psi(\sigma)=1$ ), then $\left(\left(\alpha_{i}^{\sigma}\right)^{\psi(\sigma)} / \alpha_{i}\right)^{2^{k}}=\beta_{i}^{\sigma} / \beta_{i}=1$, so

$$
\left(\alpha_{2}^{\sigma}\right)^{\psi(\sigma)} / \alpha_{2}=\alpha_{2}^{\sigma} / \alpha_{2}=\left(\left(x^{\sigma} \alpha_{1}^{\sigma}\right) /\left(x \alpha_{1}\right)\right)^{n}=\left(\alpha_{1}^{\sigma} / \alpha_{1}\right)^{n}=\left(\left(\alpha_{1}^{\sigma}\right)^{\psi(\sigma)} / \alpha_{1}\right)^{n} \in \boldsymbol{\mu}_{2^{k}},
$$

since $x \in K_{v}$. By hypothesis (iii) we have

$$
\tau_{E_{1} / K_{v}}\left(\boldsymbol{\mu}_{2^{k}}\right)=\mathcal{C}_{E_{1} / K_{v}}\left[2^{k}\right]=\mathcal{C}_{E_{2} / K_{v}}\left[2^{k}\right]=\tau_{E_{2} / K_{v}}\left(\boldsymbol{\mu}_{2^{k}}\right)
$$

inside $E_{1}\left[2^{k}\right]=E_{2}\left[2^{k}\right]$, and $n$ is odd, so $c_{1}$ and $c_{2}$ generate the same subgroup of $H^{1}\left(K_{v}, E\left[2^{k}\right]\right)$.

If $\sigma$ does not fix $L$ (i.e., $\psi(\sigma)=-1$ ), then $\left(\alpha_{i}^{\sigma}\right)^{\psi(\sigma)} / \alpha_{i}=1 /\left(\alpha_{i}^{\sigma} \alpha_{i}\right)$, and $\left(\alpha_{1}^{\sigma} \alpha_{1}\right)^{2^{k}}=\beta_{1}^{\sigma} \beta_{1}=N_{L / K_{v}}\left(\beta_{1}\right)=q_{1}$, so by Lemma 5.7(ii) applied with $\pi_{1}=\alpha_{i}^{\sigma} \alpha_{i}$ we have

$$
\begin{aligned}
& \tau_{E_{1} / K_{v}}\left(\left(\alpha_{1}^{\sigma}\right)^{\psi(\sigma)} / \alpha_{1}\right)=\tau_{E_{2} / K_{v}}\left(x^{-2}\left(\alpha_{1}^{\sigma}\right)^{\psi(\sigma)} / \alpha_{1}\right) \\
& \quad=\tau_{E_{2} / K_{v}}\left(\left(\left(\alpha_{2}^{\sigma}\right)^{\psi(\sigma)} / \alpha_{2}\right)^{n}\right)=n \tau_{E_{2} / K_{v}}\left(\left(\alpha_{2}^{\sigma}\right)^{\psi(\sigma)} / \alpha_{2}\right) .
\end{aligned}
$$

Thus $c_{1}=n c_{2}$ in $H^{1}\left(K_{v}, E\left[2^{k}\right]\right)$, and $n$ is odd, so $c_{1}$ and $c_{2}$ generate the same subgroup. Thus image $\left(\kappa_{E_{1} / K_{v}}\right)=\operatorname{image}\left(\kappa_{E_{2} / K_{v}}\right)$ in Case 4.

Case 5: $v \mid p, p>2, E_{1}$ and $E_{2}$ have good reduction at $v$, and the isomorphism $E_{1}\left[p^{k}\right] \cong E_{2}\left[p^{k}\right]$ extends to an isomorphism $\mathcal{E}_{1}\left[p^{k}\right] \cong \mathcal{E}_{2}\left[p^{k}\right]$. In this case Proposition 5.8 shows that image $\left(\kappa_{E_{1} / K_{v}}\right)=\operatorname{image}\left(\kappa_{E_{2} / K_{v}}\right)$. More generally, suppose $\chi \in$ $\mathcal{X}\left(K_{v}\right)$, and let $L$ be the quadratic extension of $K_{v}$ cut out by $\chi$. Then Proposition 5.8 shows that image $\left(\kappa_{E_{1} / L}\right)=\operatorname{image}\left(\kappa_{E_{2} / L}\right)$, and this isomorphism preserves the natural action of $\operatorname{Gal}\left(L / K_{v}\right)$. Let $\sigma$ denote the non-trivial element of $\operatorname{Gal}\left(L / K_{v}\right)$, and for every $\mathbf{Z}_{p}\left[\operatorname{Gal}\left(L / K_{v}\right)\right]$-module $M$, define $M^{-}:=\left\{m \in M: m^{\sigma}=-m\right\}$. Since $p>2$, one verifies easily using the Hochschild-Serre spectral sequence that for $i=1,2$ we have

$$
\begin{aligned}
E_{i}^{\chi}\left(K_{v}\right) / p^{k} E_{i}^{\chi}\left(K_{v}\right)= & \left(E_{i}(L) / p^{k} E_{i}(L)\right)^{-}, \quad H^{1}\left(K_{v}, E_{i}^{\chi}\left[p^{k}\right]\right)=H^{1}\left(L, E_{i}\left[p^{k}\right]\right)^{-}, \\
& \operatorname{image}\left(\kappa_{E_{i}^{\chi} / K_{v}}\right)=\operatorname{image}\left(\kappa_{E_{i} / L}\right)^{-}
\end{aligned}
$$


and we conclude that image $\left(\kappa_{E_{1}^{\chi} / K_{v}}\right)=\operatorname{image}\left(\kappa_{E_{2}^{\chi} / K_{v}}\right)$.

Thus image $\left(\kappa_{E_{1}^{\chi} / K_{v}}\right)=\operatorname{image}\left(\kappa_{E_{2}^{\chi} / K_{v}}\right)$ in (5) for every $v$ and every $\chi$, and we conclude that $E_{1}$ and $E_{2}$ are $p$-Selmer companions over $K$.

If $F$ is a finite extension of $K$, then $E_{1}$ and $E_{2}$ satisfy the hypotheses of Theorem 6.1 over $F$ as well. Applying the proof above with $F$ in place of $K$ shows that $E_{1}$ and $E_{2}$ are $p$-Selmer companions over $F$.

Proof of Theorem 3.1. Theorem 3.1 is identical to Theorem 6.1 except for the second part of hypothesis (iv). Suppose $k=1, \mathfrak{p} \mid p, E_{1}, E_{2}$ have good reduction at $\mathfrak{p}$, and the ramification $e(\mathfrak{p} / p)<p-1$ (so in particular $p>2$ ). By Raynaud's theorem $[20, \S 3.5 .5]$, the group scheme $\mathcal{E}_{i}[p]$ is determined by the Galois module $E_{i}[p]$. Hence in this case the isomorphism $E_{1}[p] \cong E_{2}[p]$ necessarily extends to an isomorphism $\mathcal{E}_{1}[p] \cong \mathcal{E}_{2}[p]$. Thus if hypotheses (i) and (iv) of Theorem 3.1 are satisfied, then hypothesis (iv) of Theorem 6.1 is satisfied. In this way Theorem 3.1 follows from Theorem 6.1

\section{Additional QUeStions AND REMARKS}

In this section we discuss some related questions, including partial converses to Theorem 3.1. Fix a number field $K$.

\subsection{The number of companions.}

Proposition 7.1. Fix an elliptic curve $E_{1}$ over $K$, and a prime power $p^{k}>2$. There are only finitely many elliptic curves $E_{2} / K$ (up to isomorphism) satisfying hypotheses (i) and (ii) of Theorem 3.1 .

Proof. Let

$$
\Sigma=\left\{\text { primes } \mathfrak{l} \text { of } K: E_{1} \text { has bad reduction at } \mathfrak{l}\right\} \cup\{\mathfrak{l}: \mathfrak{l} \mid p\} .
$$

Suppose that $E_{1}$ and $E_{2}$ satisfy (i) and (ii) of Theorem 3.1 and $\mathfrak{l}$ is a prime of $K$ not dividing $p$. If $E_{2}$ has potentially multiplicative reduction at $\mathfrak{l}$, then $\mathfrak{l} \in S_{2}=S_{1} \subset \Sigma$. If $E_{2}$ has additive, potentially good reduction at $\mathfrak{l}$, then so does $E_{1}$ by Lemma 5.3 . so $\mathfrak{l} \in \Sigma$. Thus $E_{2}$ has good reduction at all primes outside of $\Sigma$. By a theorem of Shafarevich [25], there are only finitely many such elliptic curves $E_{2} / K$.

\subsection{Selmer parity companions.}

Definition 7.2. If $p$ is a prime, we will say that two elliptic curves $E_{1}, E_{2}$ over $K$ are $p$-Selmer parity companions if for every quadratic character $\chi$ of $K$ we have

$$
\operatorname{dim}_{\mathbf{F}_{p}} \operatorname{Sel}_{p}\left(E_{1}^{\chi} / K\right) \equiv \operatorname{dim}_{\mathbf{F}_{p}} \operatorname{Sel}_{p}\left(E_{2}^{\chi} / K\right) \quad(\bmod 2) .
$$

Remark 7.3. Clearly, if $E_{1}$ and $E_{2}$ are $p$-Selmer companions, then they are also p-Selmer parity companions.

Definition 7.4. For every elliptic curve $E / K$, define a set of rational primes $T(E):=\left\{p: E^{\chi}(K)[p] \neq 0\right.$ for at least one quadratic character $\chi$ of $\left.K\right\}$.

Lemma 7.5. For every elliptic curve $E / K$, the set $T(E)$ is finite.

Proof. This is [6, Proposition 1] when $K=\mathbf{Q}$, and [16, Lemma 5.5] in general.

Recall that the Shafarevich-Tate conjecture asserts that the Shafarevich-Tate groups of all elliptic curves over $K$ are finite. 
Proposition 7.6. Suppose that either $K=\mathbf{Q}$, or the Shafarevich-Tate conjecture holds for $K$. If $E_{1}, E_{2}$ are elliptic curves over $K$, then $E_{1}$ and $E_{2}$ are $p$-Selmer parity companions for one prime $p \notin T\left(E_{1}\right) \cup T\left(E_{2}\right)$ if and only if they are $p$-Selmer parity companions for every prime $p \notin T\left(E_{1}\right) \cup T\left(E_{2}\right)$.

Proof. Suppose first that $K=\mathbf{Q}$, and for every elliptic curve $E / \mathbf{Q}$ define

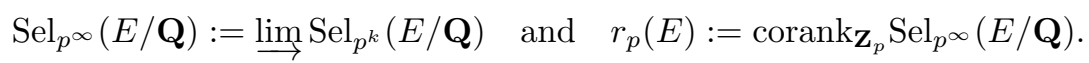

Let $D$ denote the maximal divisible subgroup of $\operatorname{Sel}_{p}(E / \mathbf{Q})$. If $E(K)[p]=0$, then $\operatorname{Sel}_{p}(E / \mathbf{Q})=\operatorname{Sel}_{p \infty}(E / \mathbf{Q})[p]$, and so the Cassels pairing shows that

$$
\operatorname{dim}_{\mathbf{F}_{p}} \operatorname{Sel}_{p}(E / \mathbf{Q})=r_{p}(E)+\operatorname{dim}_{\mathbf{F}_{p}}\left(\operatorname{Sel}_{p}(E / \mathbf{Q}) / D\right)[p] \equiv r_{p}(E) \quad(\bmod 2) .
$$

It is proved in [5] that $(-1)^{r_{p}(E)}=w(E)$, the global root number of the $L$-function of $E$. Thus the parity of $\operatorname{dim}_{\mathbf{F}_{p}} \operatorname{Sel}_{p}(E / \mathbf{Q})$ is independent of $p \notin T(E)$ in this case.

Now let $K$ be arbitrary. If the Shafarevich-Tate group of an elliptic curve $E$ over $K$ is finite, then the Cassels pairing shows that

$$
\operatorname{dim}_{\mathbf{F}_{p}} \operatorname{Sel}_{p}(E / K) \equiv \operatorname{rank}(E(K))+\operatorname{dim}_{\mathbf{F}_{p}} E(K)[p] \quad(\bmod 2) .
$$

Therefore in this case also, we have that the parity of $\operatorname{dim}_{\mathbf{F}_{p}} \operatorname{Sel}_{p}(E / K)$ is independent of $p \notin T(E)$. The proposition follows easily.

Example 7.7. Consider the curves $E_{1}=1026 N 1$ and $E_{2}=1026 O 1$ over $\mathbf{Q}$ of the Introduction. Then $E_{1}$ and $E_{2}$ are 7-Selmer companions, so they are 7-Selmer parity companions.

We claim that $T\left(E_{1}\right)$ is empty and $T\left(E_{2}\right)=\{3\}$. Let $\rho_{i, p}: G_{\mathbf{Q}} \rightarrow \operatorname{Aut}\left(E_{i}[p]\right)$ be the $\bmod p$ representation attached to $E_{i}$. For every $p \leq 4861$ one can check using Sage [22] that $\rho_{i, p}$ is surjective except when $p=3$ and $i=2$, and for $p>4861$ we have that $\rho_{i, p}$ is surjective by [4, Theorem 2]. When $\rho_{i, p}$ is surjective, no quadratic twist $E_{i}^{\chi}$ will have a rational point of order $p$ because the $\bmod p$ representation attached to $E_{i}^{\chi}$ is $\rho_{i, p} \otimes \chi$, which is irreducible. This proves the claim.

Thus Proposition [7.6 shows that $E_{1}$ and $E_{2}$ are $p$-Selmer parity companions for every prime $p \neq 3$. In fact, they are not 3-Selmer parity companions because $\operatorname{Sel}_{3}\left(E_{1} / \mathbf{Q}\right)=0$ and $\operatorname{dim}_{\mathbf{F}_{3}} \operatorname{Sel}_{3}\left(E_{2} / \mathbf{Q}\right)=1$ (note that $E_{2}$ has a rational point of order 3$)$.

This shows that $p$-Selmer parity companions need not have the same Galois action on their $p$-torsion points.

We can use the methods and results of [11] (see also [12]) to determine when two elliptic curves are 2-Selmer parity companions. Fix $p=2$.

For every elliptic curve $E / K$ and place $v$ of $K$, let $\omega_{E, v}: \mathcal{X}\left(K_{v}\right) \rightarrow\{ \pm 1\}$ be the map (of sets, not in general a homomorphism) given by [11, Definition 7.1]. Namely, $\omega_{E, v}(\chi):=(-1)^{d_{v}(\chi)} \chi\left(\Delta_{E}\right)$ where

$$
d_{v}(\chi):=\operatorname{dim}_{\mathbf{F}_{2}} \operatorname{image}\left(\kappa_{E / K_{v}}\right) /\left(\operatorname{image}\left(\kappa_{E / K_{v}}\right) \cap \operatorname{image}\left(\kappa_{E \chi / K_{v}}\right)\right) \text {, }
$$

$\Delta_{E}$ is the discriminant of $E$, and we view $\chi$ as a character of $K_{v}^{\times}$via local class field theory.

Theorem 7.8. Suppose $E_{1}, E_{2}$ are elliptic curves over $K$, and $\Sigma$ is a finite set of places of $K$ containing all places above 2 and $\infty$, and all primes where either $E_{1}$ or $E_{2}$ has bad reduction. Then $E_{1}$ and $E_{2}$ are 2 -Selmer parity companions if and only if $\operatorname{dim}_{\mathbf{F}_{2}} \operatorname{Sel}_{2}\left(E_{1} / K\right) \equiv \operatorname{dim}_{\mathbf{F}_{2}} \operatorname{Sel}_{2}\left(E_{2} / K\right)(\bmod 2)$ and $\omega_{E_{1}, v}=\omega_{E_{2}, v}$ for every $v \in \Sigma$. 
Proof. This follows directly from [11, Proposition 7.2].

The next corollary comes close to showing that hypothesis (ii) of Theorem 3.1 is a necessary condition.

Corollary 7.9. Suppose $E_{1}$ and $E_{2}$ are 2-Selmer parity companions, and $\mathfrak{l}$ is a prime of $K$ not dividing 2 . Then:

(i) $E_{1}$ has split multiplicative reduction at $\mathfrak{l}$ if and only if $E_{2}$ does,

(ii) $E_{1}$ has potentially multiplicative reduction at $\mathfrak{l}$ if and only if $E_{2}$ does.

In particular if $S_{i}$ is the set of primes where $E_{i}$ has potentially multiplicative reduction as in Theorem 3.1, and $\Sigma_{2}:=\{\mathfrak{l}: \mathfrak{l} \mid 2\}$, then $S_{1} \cup \Sigma_{2}=S_{2} \cup \Sigma_{2}$.

Proof. By Theorem 7.8, we have $\omega_{E_{1}, \mathfrak{l}}=\omega_{E_{2}, \mathfrak{l}}$. Both assertions now follow directly from [11, Proposition 7.9].

Remark 7.10. When $K=\mathbf{Q}$, Corollary 7.9 is closely related to Theorem 1 of 9$]$.

Corollary 7.11. Suppose $E_{1}$ and $E_{2}$ are 2 -Selmer parity companions, and $E_{1}[4] \cong$ $E_{2}[4]$ as $G_{K}$-modules. If $\mathfrak{l}$ is a prime of $K$ not dividing 2 , then $E_{1}$ has good (resp., additive, resp., multiplicative) reduction at $\mathfrak{l}$ if and only if $E_{2}$ does.

Proof. Combining Lemma 5.3 and Corollary 7.9 , we get that

- $E_{1}$ has good reduction at $\mathfrak{l}$ if and only if $E_{2}$ does,

- $E_{1}$ has additive, potentially good reduction at $\mathfrak{l}$ if and only if $E_{2}$ does,

- $E_{1}$ has potentially multiplicative reduction at $\mathfrak{l}$ if and only if $E_{2}$ does.

In the last case, Lemma 5.7 shows that $E_{1}$ and $E_{2}$ have the same splitting character over $K_{\mathfrak{l}}$, so $E_{1}$ has multiplicative reduction at $\mathfrak{l}$ if and only if $E_{2}$ does. This completes the proof.

\subsection{Selmer near-companions.}

Definition 7.12. We will say that two elliptic curves $E_{1}, E_{2}$ over $K$ are $n$-Selmer near-companions if there is a constant $C=C\left(E_{1}, E_{2}, K\right)$ such that for every $\chi \in$ $\mathcal{X}(K)$ there is an abelian group $A_{\chi}$ and homomorphisms $\operatorname{Sel}_{n}\left(E_{1}^{\chi} / K\right) \rightarrow A_{\chi}$ and $\operatorname{Sel}_{n}\left(E_{2}^{\chi} / K\right) \rightarrow A_{\chi}$ with kernel and cokernel of order at most $C$.

Note that $E_{1}$ and $E_{2}$ are $n$-Selmer companions if and only if Definition 7.12 is satisfied with $C=1$.

Theorem 7.13. Suppose $E_{1}$ and $E_{2}$ are elliptic curves over $K$. Let $m=p^{k+1}$ if $p \leq 3$, and $m=p^{k}$ if $p>3$. Suppose further that there is a $G_{K}$-isomorphism $E_{1}[m] \cong E_{2}[m]$. Then $E_{1}$ and $E_{2}$ are $p^{k}$-Selmer near-companions over every finite extension $F$ of $K$.

Proof. If $E$ is an elliptic curve over $K$ and $\Sigma$ is a finite set of places of $K$, define

$$
\operatorname{Sel}_{p^{k}}^{\Sigma}(E / K):=\operatorname{ker}\left(H^{1}\left(K, E\left[p^{k}\right]\right) \longrightarrow \bigoplus_{v \notin \Sigma} H^{1}\left(K_{v}, E\left[p^{k}\right]\right) / \operatorname{image}\left(\kappa_{E / K_{v}}\right)\right) .
$$

Then the definitions yield an exact sequence

$$
0 \longrightarrow \operatorname{Sel}_{p^{k}}(E / K) \longrightarrow \operatorname{Sel}_{p^{k}}^{\Sigma}(E / K) \longrightarrow \bigoplus_{v \in \Sigma} H^{1}\left(K_{v}, E\left[p^{k}\right]\right) / \operatorname{image}\left(\kappa_{E / K_{v}}\right) .
$$

Now let $\Sigma$ be the finite set of places of $K$ dividing $p \Delta_{E_{1}} \Delta_{E_{2}} \infty$. Using the given $G_{K}$-isomorphism to identify $E_{1}\left[p^{k}\right]$ with $E_{2}\left[p^{k}\right]$, Cases 2 and 3 of the proof of Theorem 6.1 show that if $v \notin \Sigma$, then image $\left(\kappa_{E_{1}^{\chi} / K_{v}}\right)=\operatorname{image}\left(\kappa_{E_{2}^{\chi} / K_{v}}\right)$ for 
every $\chi \in \mathcal{X}(K)$. Therefore $\operatorname{Sel}_{p^{k}}^{\Sigma}\left(E_{1}^{\chi} / K\right)=\operatorname{Sel}_{p^{k}}^{\Sigma}\left(E_{2}^{\chi} / K\right)$ inside $H^{1}\left(K, E_{1}\left[p^{k}\right]\right)=$ $H^{2}\left(K, E\left[p^{k}\right]\right)$, and we define $A_{\chi}$ to be this common group. Now if we let

$$
C:=\prod_{v \in \Sigma} \sup _{\chi \in \mathcal{X}\left(K_{v}\right)}\left|H^{1}\left(K_{v}, E_{1}^{\chi}\left[p^{k}\right]\right)\right|
$$

then (6) applied to $E_{1}^{\chi}$ and $E_{2}^{\chi}$ shows that $E_{1}$ and $E_{2}$ are $p^{k}$-Selmer near-companions over $K$. The same proof applies if we replace $K$ by any finite extension.

Example 7.14. Take $K=\mathbf{Q}$ and $p^{k}=2$, and let $E_{1}$ and $E_{2}$ be the elliptic curves $26 A 1$ and $598 B 1$ in 2 :

$$
\begin{aligned}
& E_{1}: y^{2}+x y+y=x^{3}-5 x-8, \\
& E_{2}: y^{2}+x y=x^{3}-x^{2}+44 x+496 .
\end{aligned}
$$

The method of A.1 below allows us to verify that there is a $G_{\mathbf{Q}}$-isomorphism $E_{1}[4] \cong E_{2}[4]$, so $E_{1}$ and $E_{2}$ are 2-Selmer near-companions by Theorem 7.13. Using a little more care, we can show that $\operatorname{Sel}_{p^{k}}^{\Sigma}\left(E_{1}^{\chi} / K\right)=\operatorname{Sel}_{p^{k}}^{\Sigma}\left(E_{2}^{\chi} / K\right)$ for every $\chi$, with $\Sigma=\{23\}$. In the exact sequences (6) for $E_{1}^{\chi}$ and $E_{2}^{\chi}$ with this $\Sigma$, the right-hand group has order $\left[E_{1}\left(\mathbf{Q}_{23}\right): 2 E_{1}\left(\mathbf{Q}_{23}\right)\right]=2$, independent of $\chi$. It follows that for every quadratic character $\chi$ of $G_{\mathbf{Q}}$ we have

$$
\operatorname{dim}_{\mathbf{F}_{2}} \operatorname{Sel}_{2}\left(E_{1}^{\chi} / \mathbf{Q}\right)-\operatorname{dim}_{\mathbf{F}_{2}} \operatorname{Sel}_{2}\left(E_{2}^{\chi} / \mathbf{Q}\right) \in\{-1,0,1\} .
$$

All three values $-1,0,1$ occur. (Note that $E_{1}$ has good reduction at 23 and $E_{2}$ has multiplicative reduction, so hypothesis (ii) of Theorem 3.1 does not hold.)

It is natural to make the following conjecture.

Conjecture 7.15. If $n \geq 1$ and $E_{1}, E_{2}$ are $n$-Selmer near-companions over $K$, then $E_{1}[n]$ is $G_{K}$-isomorphic to $E_{2}[n]$.

\section{Appendix A. Checking that $E_{1}[m] \cong E_{2}[m]$}

Suppose $E_{1}$ and $E_{2}$ are elliptic curves over $K$. In this Appendix we discuss two methods for verifying that there is a $G_{K}$-isomorphism $E_{1}[m] \cong E_{2}[m]$.

A.1. Universal families. If $m=3$, 4, or 5, then [21, Theorem 4.1], 26, Theorem 4.1], and [21, Theorem 5.1], respectively, give explicit models

$$
\tilde{E}_{t}: y^{2}=x^{3}+a(t) x+b(t), \quad a(t), b(t) \in K[t]
$$

for the family of all elliptic curves $E / K$ with $E[m]$ symplectically $G_{K}$-isomorphic to $E_{1}[m]$. (A symplectic isomorphism is one that preserves the Weil pairings.) In other words, there is a symplectic $G_{K}$-isomorphism $E_{1}[\mathrm{~m}] \cong E_{2}[\mathrm{~m}]$ if and only if there is an $s \in K$ such that the specialization $\tilde{E}_{s}$ is isomorphic over $K$ to $E_{2}$.

To test this, we can simply compute the $j$-invariant of $\tilde{E}_{t}$

$$
j(t):=1728 \frac{a(t)^{3}}{4 a(t)^{3}+27 b(t)^{2}} \in K(t),
$$

and then find (the finite set of) all zeros in $K$ of the rational function $j(t)-j\left(E_{2}\right)$. For each zero $s \in K$, we have that $\tilde{E}_{s}$ is an elliptic curve over $K$ with $j\left(\tilde{E}_{s}\right)=$ $j(s)=j\left(E_{2}\right)$, and it is then a simple matter to test whether $\tilde{E}_{s}$ is isomorphic to $E_{2}$ over $K$. If it is, then $E_{1}[m]$ is (symplectically) $G_{K}$-isomorphic to $E_{2}[m]$. But if for every zero $s \in K$ of $j(t)-j\left(E_{2}\right)$ we have that $\tilde{E}_{s}$ is not isomorphic over $K$ to $E_{2}$, then there is no symplectic $G_{K}$-isomorphism $E_{1}[m] \cong E_{2}[m]$. 
In the case $m=4$, one can show that there is a $G_{K}$-isomorphism $E_{1}[4] \cong E_{1}^{\Delta}[4]$ that is not symplectic, where $E_{1}^{\Delta}$ is the quadratic twist of $E_{1}$ by its discriminant $\Delta$. It follows that if $E_{2}$ [4] is $G_{K}$-isomorphic to $E_{1}$ [4], then $E_{2}[4]$ is symplectically $G_{K}$-isomorphic either to $E_{1}[4]$ or to $E_{1}^{\Delta}[4]$, so we can use the argument above to test for all $G_{K}$-isomorphisms, not just the symplectic ones.

Proof of Proposition 4.7. Let $E_{1}$ and $E_{2}$ be as in Example 4.6. Then $E_{1}$ has the short Weierstrass model $y^{2}=x^{3}-5616 x-494208$. Applying [21, Theorem 5.1] with $a=-5616$ and $b=-494208$ gives an explicit model of the elliptic curve $\tilde{E}_{t} / \mathbf{Q}(t)$ of (7) parametrizing the family of all elliptic curves $E / K$ with $E[5]$ symplectically $G_{\mathbf{Q}^{-i s o m o r p h i c}}$ to $E_{1}[5]$. If we set $j(t):=j\left(\tilde{E}_{t}\right)$, then we compute that $t=-9 / 22$ is a zero of the rational function $j(t)-j\left(E_{2}\right)$. Specializing $\tilde{E}_{t}$ at $t=-9 / 22$ gives a Weierstrass model of an elliptic curve isomorphic over $\mathbf{Q}$ to $E_{2}$. Thus there is a (symplectic) $G_{\mathbf{Q}^{-}}$isomorphism $E_{1}[5] \cong E_{2}[5]$.

A.2. Constructing an explicit isomorphism. Suppose $m=p^{n}$. Let $f_{i}(x) \in$ $K[x]$ be the polynomial of degree $d:=\left(p^{2 n}-p^{2 n-2}\right) / 2$ whose roots are the $x$ coordinates of the points in $E_{i}\left[p^{n}\right]-E_{i}\left[p^{n-1}\right]$. Suppose further that $f_{1}$ and $f_{2}$ are irreducible; this is equivalent to the requirement that $G_{K}$ acts transitively on $\left(E_{i}\left[p^{n}\right]-E_{i}\left[p^{n-1}\right]\right) /\{ \pm 1\}$ for $i=1,2$.

Fix a root $\alpha_{1}$ of $f_{1}$. Suppose that $f_{2}$ also has at least one root $\alpha_{2} \in K\left(\alpha_{1}\right)$. (If $E_{1}\left[p^{n}\right] \cong E_{2}\left[p^{n}\right]$, then, this will necessarily be the case.) Fix such a root and call it $\alpha_{2}$. Then $K\left(\alpha_{2}\right)=K\left(\alpha_{1}\right)$, so there is a unique polynomial $\phi(x) \in K[x]$ of degree less than $d$ such that $\phi\left(\alpha_{1}\right)=\alpha_{2}$. Since $\alpha_{1}$ is a root of $f_{2} \circ \phi$, we have that $f_{1}$ divides $f_{2} \circ \phi$, so $\phi$ maps all roots of $f_{1}$ to roots of $f_{2}$.

Fix a prime $\mathfrak{l}$ of $K$ such that $E_{1}$ and $E_{2}$ have good reduction at $\mathfrak{l}$, and $\mathfrak{l}$ splits completely in $K\left(E_{1}\left[p^{n}\right]\right) / K$. Fix a prime of $K\left(E_{1}\left[p^{n}\right]\right)$ above $\mathfrak{l}$. If $\mathbf{F}_{\mathfrak{l}}$ denotes the residue field of $\mathfrak{l}$, this choice gives us a reduction isomorphism

$$
\pi: E_{1}(\bar{K})\left[p^{n}\right] \stackrel{\sim}{\rightarrow} E_{1}\left(\mathbf{F}_{\mathfrak{l}}\right)\left[p^{n}\right] .
$$

Note that $\phi$ also maps all roots of $f_{1}$ in $\mathbf{F}_{\mathfrak{l}}$ to roots of $f_{2}$ in $\mathbf{F}_{l}$.

Fix a basis $P_{1}, P_{2}$ of $E_{1}\left(\mathbf{F}_{\mathfrak{l}}\right)\left[p^{n}\right]$. Let $Q_{1}, Q_{2} \in E_{1}\left(\mathbf{F}_{\mathfrak{l}}\right)\left[p^{n}\right]$ be points such that $x\left(Q_{i}\right)=\phi\left(x\left(P_{i}\right)\right)$. Define a group homomorphism $\varphi: E_{1}\left(\mathbf{F}_{\mathfrak{l}}\right)\left[p^{n}\right] \rightarrow E_{2}\left(\mathbf{F}_{\mathfrak{l}}\right)\left[p^{n}\right]$ by

$$
\varphi\left(a P_{1}+b P_{2}\right)=a Q_{1}+b Q_{2} \quad \text { for } a, b \in \mathbf{Z} / p^{n} \mathbf{Z} .
$$

Using the reduction isomorphism $\pi$, we can lift $\varphi$ to a group homomorphism $E_{1}\left[p^{n}\right] \rightarrow E_{2}\left[p^{n}\right]$, which we also denote by $\varphi$. Consider the diagram

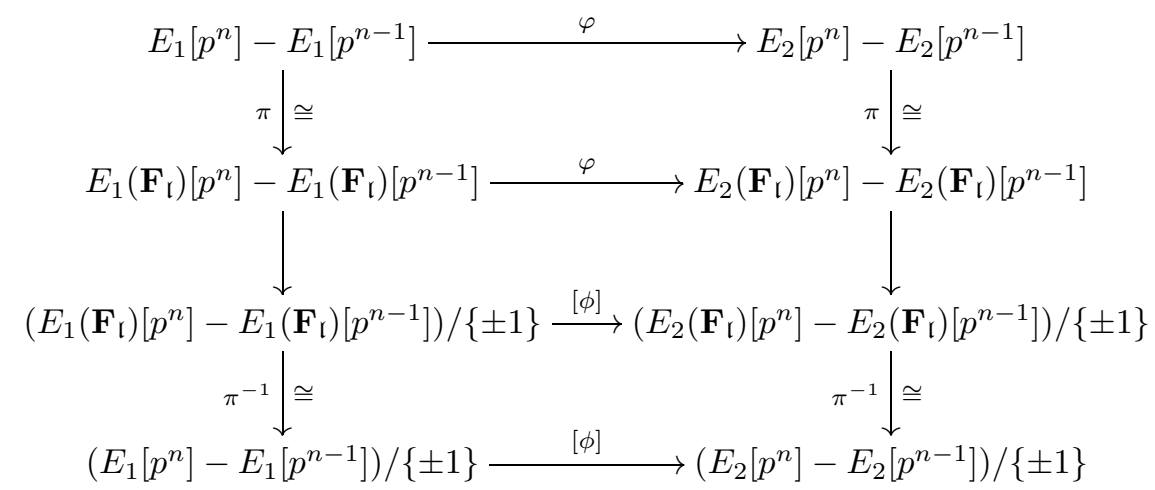


where $[\phi]$ denotes the map induced by applying the polynomial $\phi$ to the $x$-coordinates. The upper and lower squares are commutative, with vertical isomorphisms.

Let $S$ be the set of places of $K$ where at least one of $E_{1}\left[p^{n}\right], E_{2}\left[p^{n}\right]$ is ramified, and $\mathcal{X}(K, S) \subset \mathcal{X}(K)$ the (finite) subgroup of characters unramified outside of $S$.

Lemma A.1. Suppose that the center square of (8) is commutative. Then:

(i) $\varphi$ is a group isomorphism,

(ii) there is a quadratic character $\psi \in \mathcal{X}(K, S)$ such that $\varphi\left(P^{\sigma}\right)=\psi(\sigma) \varphi(P)^{\sigma}$ for every $P \in E_{1}\left[p^{n}\right]$ and $\sigma \in G_{K}$.

Proof. If the center square of (8) is commutative, then the entire diagram is commutative. Since $\phi \in K[x]$, the bottom map $[\phi]$ is $G_{K}$-equivariant. Since $f_{2}$ is irreducible, $G_{K}$ acts transitively on $\left(E_{2}\left[p^{n}\right]-E_{2}\left[p^{n-1}\right]\right) /\{ \pm 1\}$. Hence the bottom map $[\phi]$ is surjective, so the top map $\phi$ is surjective, and (i) follows.

It also follows from the commutativity of (8) and the $G_{K}$-equivariance of $[\phi]$ that

$$
\varphi\left(P^{\sigma}\right)= \pm \varphi(P)^{\sigma} \quad \text { for every } P \in E_{1}\left[p^{n}\right] \text { and } \sigma \in G_{K} .
$$

For every $\sigma \in G_{K}$, the sets

$$
\left\{P \in E_{1}\left[p^{n}\right]: \varphi\left(P^{\sigma}\right)=\varphi(P)^{\sigma}\right\}, \quad\left\{P \in E_{1}\left[p^{n}\right]: \varphi\left(P^{\sigma}\right)=-\varphi(P)^{\sigma}\right\}
$$

are subgroups of $E\left[p^{n}\right]$. It follows from (9) that the union of these two subgroups is $E\left[p^{n}\right]$, and therefore one of them must be all of $E\left[p^{n}\right]$. Thus for every $\sigma$ we can define $\psi(\sigma)= \pm 1$ so that $\varphi\left(P^{\sigma}\right)=\psi(\sigma) \varphi(P)^{\sigma}$ for every $P \in E_{1}\left[p^{n}\right]$. One sees easily that $\psi \in \mathcal{X}(K)$, and $\psi$ is necessarily unramified outside $S$, so $\psi \in \mathcal{X}(K, S)$.

Suppose now that the center square of (8) is commutative. Let $\psi \in \mathcal{X}(K, S)$ be the character of Lemma A.1(ii). Then by Lemma A.1, $\varphi$ induces an isomorphism $E_{1}\left[p^{n}\right] \stackrel{\sim}{\rightarrow} E_{2}^{\psi}\left[p^{n}\right]$, where $E_{2}^{\psi}$ is the quadratic twist of $E_{2}$ by $\psi$. We would like to verify that $\psi$ must be the trivial character.

Fix a basis $\chi_{1}, \ldots, \chi_{t}$ of the $\mathbf{F}_{2}$-vector space $\mathcal{X}(K, S)$. Suppose that for every $i$, $1 \leq i \leq t$, we can find a prime $\mathfrak{q}_{i}$ of $K, \mathfrak{q}_{i} \notin S$, such that

- $\chi_{i}\left(\mathfrak{q}_{i}\right)=-1, \chi_{j}\left(\mathfrak{q}_{i}\right)=1$ if $j \neq i$,

- the traces of Frobenius of $\mathfrak{q}_{i}$ on $E_{1}\left[p^{n}\right]$ and $E_{2}\left[p^{n}\right]$ satisfy

$$
\operatorname{Trace}\left(\operatorname{Frob}_{\mathfrak{q}_{i}} \mid E_{1}\left[p^{n}\right]\right) \neq-\operatorname{Trace}\left(\operatorname{Frob}_{\mathfrak{q}_{i}} \mid E_{2}\left[p^{n}\right]\right) \text {. }
$$

Choose a non-trivial character $\chi=\prod_{i} \chi_{i}^{a_{i}} \in \mathcal{X}(K, S), a_{i} \in\{0,1\}$. If $a_{i} \neq 0$ for some $i$, then by our choice of $\mathfrak{q}_{i}$ we have $\operatorname{Trace}\left(\operatorname{Frob}_{\mathfrak{q}_{i}} \mid E_{1}\left[p^{n}\right]\right) \neq \operatorname{Trace}\left(\operatorname{Frob}_{\mathfrak{q}_{i}} \mid E_{2}^{\chi}\left[p^{n}\right]\right)$, so $E_{1}\left[p^{n}\right] \nRightarrow E_{2}^{\chi}\left[p^{n}\right]$. Hence $\psi$ must be the trivial character.

All of the steps above can be handled by either Sage [22] or PARI/GP [17]. Computing the polynomial $\phi(x)$ is the only significantly time-consuming step. Finding a prime $\mathfrak{l}$, checking the commutativity of (8), and finding primes $\mathfrak{q}_{i}$ with appropriate traces of Frobenius is very quick. (Note that the points $Q_{1}, Q_{2} \in E_{1}\left(\mathbf{F}_{\mathfrak{l}}\right)\left[p^{n}\right]$ are only defined up to multiplication by \pm 1 . If the first choice does not lead to commutativity in (8), then replacing $Q_{1}$ by $-Q_{1}$ may still work.)

Proof of Proposition 4.5. Let $E_{1}$ and $E_{2}$ be as in Example 4.4, both of conductor $1242=2 \cdot 3^{3} \cdot 23$, and $m=8$. PARI/GP computes the polynomial $\phi(x)$ of degree $\left(8^{2}-4^{2}\right) / 2=24$ in less than a minute on a modern desktop computer. We take $\mathfrak{l}:=19681$, and

$$
P_{1}:=(731,4673), P_{2}:=(3074,1044) \in E_{1}\left(\mathbf{F}_{19681}\right) .
$$


Then $P_{1}, P_{2}$ generate $E_{1}[8]$, and we compute that $\phi(731) \equiv 10530(\bmod 19681)$ and $\phi(3074) \equiv 17962(\bmod 19681)$. We define $\varphi$ as above using the points

$$
Q_{1}=(10530,9277), Q_{2}=(17962,16270) \in E_{2}\left(\mathbf{F}_{19681}\right),
$$

and we check directly that the center square of (8) commutes. Therefore Lemma A.1 shows that $\varphi: E_{1}[8] \rightarrow E_{2}^{\psi}[8]$ is a $G_{\mathbf{Q}^{-}}$equivariant isomorphism, where $\psi \in$ $\mathcal{X}(\mathbf{Q},\{\infty, 2,3,23\})$. The group $\mathcal{X}(\mathbf{Q},\{\infty, 2,3,23\})$ is generated by the characters $\chi_{-1}, \chi_{2}, \chi_{-3}, \chi_{-23}$, where $\chi_{d}$ is the quadratic character of $\mathbf{Q}(\sqrt{d}) / \mathbf{Q}$. We find the following data, where $a_{q}\left(E_{i}\right)$ is the trace of Frobenius Frob . .

\begin{tabular}{|c|c|c|c|c|c|c|}
\hline$q$ & $\chi_{-1}(q)$ & $\chi_{2}(q)$ & $\chi_{-3}(q)$ & $\chi-23(q)$ & $a_{q}\left(E_{1}\right) \quad(\bmod 8)$ & $a_{q}\left(E_{2}\right) \quad(\bmod 8)$ \\
\hline 31 & -1 & 1 & 1 & 1 & 2 & 2 \\
\hline 349 & 1 & -1 & 1 & 1 & 2 & 2 \\
\hline 233 & 1 & 1 & -1 & 1 & 2 & 2 \\
\hline 241 & 1 & 1 & 1 & -1 & 6 & 6 \\
\hline
\end{tabular}

The argument above shows that $\psi$ is the trivial character, so $\varphi: E_{1}[8] \rightarrow E_{2}[8]$ is a $G_{\mathbf{Q}^{-}}$equivariant isomorphism.

\section{REFERENCES}

[1] Spencer Bloch and Kazuya Kato, L-functions and Tamagawa numbers of motives, The Grothendieck Festschrift, Vol. I, Progr. Math., vol. 86, Birkhäuser Boston, Boston, MA, 1990, pp. 333-400. MR1086888 (92g:11063)

[2] J. E. Cremona, Algorithms for modular elliptic curves, Cambridge University Press, Cambridge, 1992. MR1201151 (93m:11053)

[3] J. W. S. Cassels, Arithmetic on curves of genus 1. VIII. On conjectures of Birch and Swinnerton-Dyer, J. Reine Angew. Math. 217 (1965), 180-199. MR0179169 (31 \#3420)

[4] Alina Carmen Cojocaru, On the surjectivity of the Galois representations associated to nonCM elliptic curves, Canad. Math. Bull. 48 (2005), no. 1, 16-31, DOI 10.4153/CMB-2005002-x. With an appendix by Ernst Kani. MR2118760 (2005k:11109)

[5] Tim Dokchitser and Vladimir Dokchitser, On the Birch-Swinnerton-Dyer quotients modulo squares, Ann. of Math. (2) 172 (2010), no. 1, 567-596, DOI 10.4007/annals.2010.172.567. MR2680426(2011h:11069)

[6] F. Gouvêa and B. Mazur, The square-free sieve and the rank of elliptic curves, J. Amer. Math. Soc. 4 (1991), no. 1, 1-23, DOI 10.2307/2939253. MR1080648 (92b:11039)

[7] D. R. Heath-Brown, The size of Selmer groups for the congruent number problem. II, Invent. Math. 118 (1994), no. 2, 331-370, DOI 10.1007/BF01231536. With an appendix by P. Monsky. MR1292115 (95h:11064)

[8] E. Kani and W. Schanz, Modular diagonal quotient surfaces, Math. Z. 227 (1998), no. 2, 337-366, DOI 10.1007/PL00004379. MR.1609061 (99a:14031)

[9] H. Kisilevsky, Rank determines semi-stable conductor, J. Number Theory 104 (2004), no. 2, 279-286, DOI 10.1016/S0022-314X(03)00157-4. MR2029506 (2005h:11137)

[10] Zev Klagsbrun, Selmer Ranks of Quadratic Twists of Elliptic Curves, ProQuest LLC, Ann Arbor, MI, 2011. Thesis (Ph.D.)-University of California, Irvine. MR2890124

[11] Z. Klagsbrun, B. Mazur and K. Rubin, Selmer ranks of quadratic twists of elliptic curves, to appear in Annals of Math.

[12] Kenneth Kramer, Arithmetic of elliptic curves upon quadratic extension, Trans. Amer. Math. Soc. 264 (1981), no. 1, 121-135, DOI 10.2307/1998414. MR597871 (82g:14028)

[13] A. Kraus and J. Oesterlé, Sur une question de B. Mazur (French), Math. Ann. 293 (1992), no. 2, 259-275, DOI 10.1007/BF01444715. MR1166121(93e:11074)

[14] Barry Mazur, Rational points of abelian varieties with values in towers of number fields, Invent. Math. 18 (1972), 183-266. MR0444670 (56 \#3020)

[15] B. Mazur, Rational isogenies of prime degree (with an appendix by D. Goldfeld), Invent. Math. 44 (1978), no. 2, 129-162, DOI 10.1007/BF01390348. MR482230(80h:14022) 
[16] B. Mazur and K. Rubin, Ranks of twists of elliptic curves and Hilbert's tenth problem, Invent. Math. 181 (2010), no. 3, 541-575, DOI 10.1007/s00222-010-0252-0. MR 2660452 (2012a:11069)

[17] PARI/GP, version 2.4.3. The PARI Group (2011) http://pari.math.u-bordeaux.fr.

[18] A. N. Parshin and Yu. G. Zarhin, Finiteness problems in Diophantine geometry, Amer. Math. Soc. Transl. 143 (1989) 35-102.

[19] Bjorn Poonen and Eric Rains, Random maximal isotropic subspaces and Selmer groups, J. Amer. Math. Soc. 25 (2012), no. 1, 245-269, DOI 10.1090/S0894-0347-2011-00710-8. MR2833483

[20] Michel Raynaud, Schémas en groupes de type $(p, \ldots, p)$ (French), Bull. Soc. Math. France 102 (1974), 241-280. MR0419467 (54 \#7488)

[21] K. Rubin and A. Silverberg, Families of elliptic curves with constant mod $p$ representations, Elliptic curves, modular forms, \& Fermat's last theorem (Hong Kong, 1993), Ser. Number Theory, I, Int. Press, Cambridge, MA, 1995, pp. 148-161. MR1363500 (96j:11078)

[22] Sage Mathematics Software, Version 4.7.2. The Sage Development Team (2011) http:// www. sagemath.org.

[23] Jean-Pierre Serre, Propriétés galoisiennes des points d'ordre fini des courbes elliptiques (French), Invent. Math. 15 (1972), no. 4, 259-331. MR0387283 (52 \#8126)

[24] Jean-Pierre Serre and John Tate, Good reduction of abelian varieties, Ann. of Math. (2) 88 (1968), 492-517. MR0236190 (38 \#4488)

[25] I. R. Šafarevič, Algebraic number fields (Russian), Proc. Internat. Congr. Mathematicians (Stockholm, 1962), Inst. Mittag-Leffler, Djursholm, 1963, pp. 163-176. MR0202709 (34 \#2569)

[26] Alice Silverberg, Explicit families of elliptic curves with prescribed mod $N$ representations, Modular forms and Fermat's last theorem (Boston, MA, 1995), Springer, New York, 1997, pp. 447-461. MR 1638488

[27] Joseph H. Silverman, The arithmetic of elliptic curves, Graduate Texts in Mathematics, vol. 106, Springer-Verlag, New York, 1986. MR817210 (87g:11070)

[28] Peter Swinnerton-Dyer, The effect of twisting on the 2-Selmer group, Math. Proc. Cambridge Philos. Soc. 145 (2008), no. 3, 513-526, DOI 10.1017/S0305004108001588. MR2464773 (2010d:11059)

[29] J. Tate, Algorithm for determining the type of a singular fiber in an elliptic pencil, Modular functions of one variable, IV (Proc. Internat. Summer School, Univ. Antwerp, Antwerp, 1972), Springer, Berlin, 1975, pp. 33-52. Lecture Notes in Math., Vol. 476. MR0393039(52 \#13850)

[30] John Tate, A review of non-Archimedean elliptic functions, Elliptic curves, modular forms, \& Fermat's last theorem (Hong Kong, 1993), Ser. Number Theory, I, Int. Press, Cambridge, MA, 1995, pp. 162-184. MR1363501 (97d:11096)

Department of Mathematics, Harvard University, Cambridge, Massachusetts 02138

E-mail address: mazur@math.harvard.edu

Department of Mathematics, University of California Irvine, Irvine, California 92697 E-mail address: krubin@math.uci.edu 